Laser Photon Rev. 2013 September 1; 7(5): . doi:10.1002/lpor.201200060.

\title{
Photoacoustic Microscopy
}

\author{
Junjie Yao and Lihong V. Wang* \\ Optical Imaging Laboratory, Department of Biomedical Engineering, Washington University in St. \\ Louis, St. Louis, MO 63130, USA
}

\begin{abstract}
Photoacoustic microscopy (PAM) is a hybrid in vivo imaging technique that acoustically detects optical contrast via the photoacoustic effect. Unlike pure optical microscopic techniques, PAM takes advantage of the weak acoustic scattering in tissue and thus breaks through the optical diffusion limit ( $\sim 1 \mathrm{~mm}$ in soft tissue). With its excellent scalability, PAM can provide highresolution images at desired maximum imaging depths up to a few millimeters. Compared with backscattering-based confocal microscopy and optical coherence tomography, PAM provides absorption contrast instead of scattering contrast. Furthermore, PAM can image more molecules, endogenous or exogenous, at their absorbing wavelengths than fluorescence-based methods, such as wide-field, confocal, and multi-photon microscopy. Most importantly, PAM can simultaneously image anatomical, functional, molecular, flow dynamic and metabolic contrasts in vivo. Focusing on state-of-the-art developments in PAM, this Review discusses the key features of PAM implementations and their applications in biomedical studies.
\end{abstract}

\section{Keywords}

Photoacoustic microscopy; multi-scale imaging; real-time imaging; functional imaging; molecular imaging; blood flow imaging; metabolic imaging; tumor imaging; gene expression imaging; brain imaging; nanoparticle imaging

\section{Introduction}

For centuries, the development of optical microscopes has revolutionized fundamental life science and clinical practice [1]. By magnifying minuscule cellular and subcellular features, optical microscopes provide a powerful tool for studying tissue components and their dynamic interactions. Its excellent imaging contrast in soft tissue has made optical microscopy the most widely used imaging modality in the biomedical community [1].

The visual power of optical microscopy relies on sharp optical focusing. Such power is rapidly reduced as photons travel deeper into biological tissue, a highly scattering medium for electromagnetic waves in the optical spectral range. When photons reach the optical diffusion limit ( $\sim \mathrm{mm}$ in tissue), they have typically undergone tens of scattering events, which randomize the photon paths and thus prevent tight focusing [2]. Although modern optical microscopic techniques have released biologists from the confines of tenmicrometer-thick ex vivo tissue slices to a world of volumetric in vivo tissue, optical microscopy is still challenged to image at depths beyond the optical diffusion limit while maintaining high resolution. For decades, engineers have made scant progress by using pure optical approaches to fight scattering. Fortunately, the emerging technique of photoacoustic

\footnotetext{
*Corresponding author: lhwang@wustl.edu.
} 
tomography (PAT) has pointed out a new direction, converting photon energy into ultrasound energy on the basis of the photoacoustic effect [3-14].

In PAT, as photons travel in tissue, some of them are absorbed by biomolecules and their energy is partially or completely converted into heat. The heat then induces an initial pressure rise, which propagates in tissue as a wideband acoustic wave [15-17]. An ultrasonic transducer or transducer array detects the acoustic wave to form an image, which maps the original optical energy deposition in the tissue. Since ultrasonic scattering by tissue $\left(\sim 1.2 \times 10^{-3} \mathrm{~mm}^{-1}\right.$ in human skin at $\left.5 \mathrm{MHz}\right)[18]$ is more than three orders of magnitude weaker than optical scattering $\left(\sim 10 \mathrm{~mm}^{-1}\right.$ in human skin at $\left.700 \mathrm{~nm}\right)$ [2], PAT can achieve fine acoustic resolution at depths beyond the optical diffusion limit. In addition, since the photoacoustic signal amplitude is proportional to the optical energy deposition, PAT is sensitive to the rich optical absorption contrast of tissue.

Several previous Review articles have given comprehensive coverage of PAT, focusing on instrumentation [4-6], contrast agents [8, 19], or biomedical applications [20-23]. The goal of this paper is to review a major implementation of PAT, photoacoustic microscopy (PAM). PAM has achieved spatial resolution ranging from sub-micrometer to submillimeter, at maximum imaging depths ranging from a few hundred micrometers to a few millimeters [3, 6]. Distinct from reconstruction-based PA computed tomography (PACT) [24-31], the other major implementation of PAT, PAM employs raster-scanning of optical and acoustic foci and forms images directly from acquired depth-resolved signals [3]. PAM maximizes its detection sensitivity by confocally aligning its optical illumination and acoustic detection. While the axial resolution of PAM is primarily determined by the imaging depth and the frequency response of the ultrasonic transducer, its lateral resolution is determined by the combined point spread function of the dual foci. PAM can be further classified into optical-resolution PAM (OR-PAM), where the optical focusing is much tighter than acoustic focusing [32], and acoustic-resolution PAM (ARPAM), where the acoustic focusing is tighter $[33,34]$. In addition, photoacoustic endoscopy (PAE) is considered as a variant of PAM for internal organ imaging, which is typically rotational scanning based. In PAM, while the depth-resolved acoustic waves render 1D PA images (Ascan), two-dimensional raster scanning generates 3D PA images (C-scan).

In this Review of PAM, we first discuss the wide length scalability, including the spatial resolution, maximum imaging depth, and detection sensitivity. Next, we introduce the recent methods that improve the imaging speed. Third, we present the rich endogenous and exogenous contrasts. Then, we highlight the diverse functionality of PAM and its representative applications. In the end, we envision future developments.

\section{Multi-scale PAM}

The scalability of PAM originates from its optical and acoustic focusing [3]. Within the optical diffusion limit, OR-PAM has a great advantage over AR-PAM in spatial resolution because the optical beam can be easily focused to a much tighter spot than the acoustic detection, owing to shorter optical wavelengths. Beyond the optical diffusion limit, however, AR-PAM can achieve better focusing, taking advantage of the weaker acoustic scattering.

\subsection{Lateral resolution}

Like confocal microscopy, OR-PAM can be implemented in reflection mode, transmission mode or double-illumination mode, depending on the application [32, 35-37]. Figure 1a shows a representative reflection-mode second-generation OR-PAM system (G2-OR-PAM) [38]. The nanosecond pulsed laser beam is tightly focused into the tissue by an optical objective. An optical-acoustic beam combiner composed of a layer of silicone oil 
sandwiched by two prisms is used for the coaxial and confocal alignment of the optical illumination and acoustic detection. The resultant ultrasound waves are first focused by a concave acoustic lens ground into the bottom of the combiner (numerical aperture: 0.44 ), and then detected by a wideband ultrasonic transducer (central frequency: $50 \mathrm{MHz}$ ) attached to the top surface of the prism. A correction lens is added to offset the optical aberration of the prism.

Since the acoustic focal diameter $(\sim 45 \mu \mathrm{m})$ is more than ten times as wide as the optical focal spot, the detection sensitivity within the optical focus is approximately uniform. Therefore, the lateral resolution of OR-PAM $R_{L, O R}$ is given by the diffraction-limited spot size of the optical focus $[3,35]$

$$
R_{L, O R}=0.51 \frac{\lambda_{0}}{N A_{0}},
$$

where $\lambda_{O}$ is the optical wavelength and $N A_{O}$ is the numerical aperture of the optical objective. The constant 0.51 reflects the full width at half maximum (FWHM) of the optical focal spot in light intensity. For a wavelength of $570 \mathrm{~nm}$ and an $N A_{\mathrm{O}}$ of 0.13 , the lateral resolution of G2-OR-PAM was experimentally determined to be $2.56 \mu \mathrm{m}$, which agrees to Eq. (1) [38]. This resolution is sufficient to resolve the cortical capillaries (diameter: 5-10 $\mu \mathrm{m}$ ) of a nude mouse with the scalp removed but skull intact (Figure 1b).

The lateral resolution of OR-PAM can be scaled down by either increasing the objective $N A_{O}$ or using a shorter excitation wavelength, with the maximum imaging depth scaled accordingly. For example, with a wavelength of $532 \mathrm{~nm}$ and an $N A_{O}$ of 1.23 implemented with water immersion, sub-wavelength PAM (SW-PAM) has achieved a lateral resolution of $220 \mathrm{~nm}$ with a maximum imaging depth of $100 \mu \mathrm{m}$ [35]. SW-PAM is able to resolve single organelles such as the melanosomes in a melanoma cell (Figure 1c) [35]. Alternatively, with a wavelength of $266 \mathrm{~nm}$ and an $N A_{O}$ of 0.2 , ultraviolet OR-PAM (UV-PAM) has achieved a lateral resolution of $0.7 \mu \mathrm{m}$ with a maximum imaging depth of $100 \mu \mathrm{m}$ [39]. UV-PAM can image the DNA and RNA in single cell nuclei without staining. The lateral resolution of OR-PAM can potentially be further improved by using an oil-immersion objective with the maximum reported $N A_{O}$ of 1.6.

Unlike OR-PAM, AR-PAM is typically implemented in reflection mode because it is mainly designed for thick objects $[33,34,40,41]$. Figure $1 d$ shows a representative dark-field ARPAM system $[33,34]$. The laser beam from a multi-mode optical fiber passes through a conical lens and provides a ring-shaped illumination area. The beam is then weakly focused into the tissue, with the focal region coaxially overlapping the focus of an ultrasonic transducer positioned in the dark center of the illumination. The acoustic focusing is achieved by a concave acoustic lens affixed to the transducer surface. Depending on the system design, a geometrically focused transducer can be used instead. The dark-field configuration can greatly reduce the surface signals (e.g., from melanin in the epidermis of human skin), and facilitate confocally aligning the optical and acoustic beams. In addition to the dark-field AR-PAM, fiber-based bright-field AR-PAM has also been developed [41]. Since the optical beam even in the absence of scattering $(>2 \mathrm{~mm})$ is much wider than the acoustic focus, the lateral resolution of AR-PAM is given by [42]

$$
R_{L, A R}=0.71 \frac{\lambda_{A}}{N A_{A}}=0.71 \frac{\nu_{A}}{N A_{A} \cdot f_{A}}
$$

where $N A_{A}$ is the numerical aperture of the ultrasonic transducer, $v_{A}$ is the speed of sound in the medium, and $\lambda_{A}$ and $f_{A}$ are the central wavelength and frequency of the PA signal, 
respectively. The constant 0.71 reflects the FWHM of the acoustic focal spot in acoustic amplitude. Note that this constant is about $\sqrt{2}$ times that of the optical focus in Eq. (1), because it is the acoustic amplitude rather than the intensity that is detected by the ultrasonic transducer. With an $N A_{A}$ of 0.44 and $f_{A}$ of $50 \mathrm{MHz}$, the dark-field AR-PAM in Figure 1d has achieved a lateral resolution of $45 \mu \mathrm{m}$ with a maximum imaging depth of $3 \mathrm{~mm}$. AR-PAM is able to resolve the major cortical blood vessels in a living mouse through intact scalp and skull (Figure 1e) [43].

As we can see from Eq. (2), the lateral resolution of AR-PAM can be scaled by varying the acoustic $f_{A}$ and $N A_{A}$. Typically, with nanosecond pulsed excitations, $f_{A}$ is largely determined by the acoustic attenuation in tissue. The frequency of the ultrasonic transducer is chosen to match the detectable signal frequency. Although high frequency waves offer finer lateral resolution, they are attenuated faster, resulting in shallower imaging depth. A typical constant accounting for the frequency-dependent acoustic attenuation is $0.6 \mathrm{~dB} \mathrm{~cm}^{-1} \mathrm{MHz}^{-1}$ in soft tissue [44]. Conversely, low frequency ultrasound results in deeper penetration. For example, by using a $5 \mathrm{MHz}$ ultrasonic transducer, deep-penetration photoacoustic macroscopy (PAMac) relaxes the lateral resolution to $560 \mu \mathrm{m}$ and extends the maximum imaging depth to a few centimeters, as shown in Figure 1f [45-47]. Since the naked eye can already discern features with $50 \mu \mathrm{m}$ resolution, PAMac is generally not classified as microscopy but is covered in this Review to demonstrate the scalability in the acoustic domain.

It should be pointed out that the lateral resolution of PAM is the finest at the depth corresponding to the focus of the optical objective for OR-PAM, or the acoustic lens for AR-PAM. At the two ends of the focal zone, the lateral resolution degrades by $\sqrt{2}$ due to diffraction. The focal zone for OR-PAM can be computed as $Z_{O R}=1.8 \lambda_{O} / N A_{O}{ }^{2}$. The focal zone for AR-PAM can be computed as $Z_{A R}=2.4 \lambda_{A} / N A_{A}{ }^{2}$. At the ends of twice the focal zone, the lateral resolution degrades by a factor of 2 . The prefactors in the two formulas are different because the former is based on light intensity and the latter is based on acoustic amplitude instead of intensity.

To maintain high lateral resolution over an extended depth range, depth scanning can be implemented at the expense of imaging speed. Alternatively, a virtual-detector-based synthetic-aperture focusing technique, combined with coherence weighting, can be used for AR-PAM [48, 49]. Furthermore, limited-diffracting Bessel beams can be used to extend the focal zone at the expense of power delivery efficiency and sidelobe suppression for ORPAM [50, 51], where strong sidelobes degrade imaging contrast.

\subsection{Axial resolution}

For axial resolution, OR-PAM and AR-PAM share the same formula, $R_{A, O R / A R}=0.88 v_{A} /$ $\Delta f_{A}$, which is based on the assumption that the PA response to a point target follows a Gaussian frequency profile [3]. $\Delta f_{A}$ is the PA signal bandwidth, which can be approximated as the detection bandwidth of the ultrasonic transducer and is often proportional to its central frequency $f_{A}$. However, this is based on the assumption that the PA signal bandwidth is much wider than the detection bandwidth. As mentioned above, high-frequency components of acoustic waves are attenuated faster than low frequency ones, and thus, PA signal bandwidth decreases with imaging depth, resulting in worse axial resolution at greater depths. Using ultrasonic transducers with a central frequency of $50 \mathrm{MHz}$ and a one-way -6 dB bandwidth of 100\%, G2-OR-PAM in Figure 1a and dark-field AR-PAM in Figure 1d have achieved the same axial resolution of $15 \mu \mathrm{m}$, despite their different lateral resolutions.

In addition to the traditional wideband piezoelectric ultrasonic transducers, a thin-film microring resonator has been used for optical detection of acoustic waves in OR-PAM [52, 
53]. The acoustic bandwidth of the microring resonator can potentially reach $1 \mathrm{GHz}$, which can lead to an axial resolution of down to $2 \mu \mathrm{m}$ at the expense of penetration. Similarly, a Fabry-Perot polymer film ultrasound sensor with an acoustic bandwidth of $100 \mathrm{MHz}$ has been explored for OR-PAM, where an axial resolution of $10 \mu \mathrm{m}$ has been achieved [54, 55].

\subsection{Maximum imaging depth}

The maximum imaging depth of OR-PAM is limited to $\sim 1 \mathrm{~mm}$ in tissue due to optical scattering (Figure 2a) [38]. By contrast, the maximum imaging depth of AR-PAM within the reach of excitation photons is primarily limited by ultrasonic attenuation, thus highly related to the optical wavelength and ultrasound frequency (Figure 2b) [33]. The optical attenuation by tissue includes both absorption and scattering [2]. Compared with absorption, scattering has a relatively weak dependence on wavelength. In general, an excitation wavelength within the tissue optical window around $700 \mathrm{~nm}$ helps achieve a deeper penetration because of the relatively low absorption of both hemoglobin and water, two major absorbers in tissue $[2,56]$. The maximum imaging depth can also be improved when the sample is illuminated from multiple directions, as demonstrated by double-illumination PAM (DI-PAM) [57]. It has been shown that the maximum imaging depth of PAM is approximately proportional to its lateral resolution [3]. So far, by varying the lateral resolution from $220 \mathrm{~nm}$ to $560 \mu \mathrm{m}$, maximum imaging depths from $100 \mu \mathrm{m}$ to $4 \mathrm{~cm}$ have been achieved by various PAM systems and PAMac.

With its high scalability, PAM can achieve a depth-to-resolution ratio (DRR) of $\sim 100$ over a wide range of depths. The DRR is defined as the ratio of the maximum imaging depth to the axial resolution, representing the number of resolution pixels in the depth dimension. As an exception, the DRR of SW-PAM or UV-PAM is much less than 100. In these cases, the strong optical focusing or the strong UV absorption reduces the imaging depth, and the available bandwidth of ultrasonic detection limits the axial resolution. As mentioned above, by using an acoustic detector with a wider bandwidth, OR-PAM can potentially achieve a better axial resolution [58].

\subsection{Detection sensitivity}

The detection sensitivity of PAM is affected by several factors, including the incident laser fluence, imaging depth, optical wavelength, absorption cross section of the target, and detection efficiency of the ultrasonic transducer [59]. When the incident intensity is much less than the saturation intensity of the target, the detection sensitivity is approximately proportional to the fluence $[60,61]$. Here, saturation intensity means the laser intensity that reduces the absorption coefficient to half of its original value. As the imaging depth increases, laser fluence decreases exponentially.

Two parameters can be used to quantify the detection sensitivity: noise-equivalent molar concentration (NEC) and noise-equivalent number of molecules per resolution voxel (NEN). The latter is the product of the former and the resolution voxel volume, associated with the noise from a voxel. Since hemoglobin is one of the most imaged contrast agents by PAM, the detection sensitivity of hemoglobin for representative PAM and PAMac systems is quantified here using in vivo data reported in the literature (Figure 3).

For a fair comparison, the incident fluence of each system is scaled to the ANSI (American National Standards Institute) limit for the skin at $570 \mathrm{~nm}\left(20 \mathrm{~mJ} / \mathrm{cm}^{2}\right.$ at the skin surface) [62]. For SW-PAM where the data was acquired at $532 \mathrm{~nm}$, the sensitivity is scaled according to the ratio of the absorption coefficients at the two wavelengths. Among the four PAM and PAMac systems, SW-PAM has the best sensitivity (NEC: $\sim 0.38 \mu \mathrm{M} ;$ NEN: $\sim 2.7 \times$ $10^{-4} \mathrm{fmol}$ ). Not surprisingly, as shown in Figure 3, both NEC and NEN increase with the 
imaging depth, another scalability of PAM. Furthermore, from the four systems shown in Figure 3, two empirical formulas can be fitted to relate NEC $(\mu \mathrm{M})$ and NEN (attomol) with imaging depth $z(\mathrm{~mm})$ :

$$
\begin{aligned}
& N E C=7.4 z^{1.3}, \\
& N E N=7.8 z^{4.6} .
\end{aligned}
$$

We note that the difference between the powers of $z$ is $\sim 3$, which suggests that the resolution voxel volume is approximately proportional to the cubic of the imaging depth.

It should be mentioned that hemoglobin is chosen here mainly because it is the only contrast agent that has been studied by all the PAM systems. The detection sensitivity can be enhanced by using exogenous contrast agents with greater absorption cross sections [63-65]. For example, an NEC of $\sim 250 \mathrm{nM}$ and an NEN of $\sim 5 \mathrm{fmol}$ have been achieved by using IRDye 800 -c(KRGDf) for PACT imaging at $\sim 2 \mathrm{~mm}$ depth in tissue phantoms at $784 \mathrm{~nm}$, with a $2.25 \mathrm{MHz}$ unfocused ultrasonic transducer [65]. While the absorption cross sections of oxy-hemoglobin and deoxy-hemoglobin at $570 \mathrm{~nm}$ are both $\sim 1.7 \times 10^{-16} \mathrm{~cm}^{2}$, the absorption cross section of IRDye 800 at $784 \mathrm{~nm}$ is $\sim 1.0 \times 10^{-15} \mathrm{~cm}^{2}$, about 6 times that of hemoglobin. Furthermore, an NEC of $\sim 17 \mathrm{pM}$ and an NEN of $\sim 6$ zeptomol (i.e., $\sim 6 \times 10^{-21}$ mol) have been demonstrated by using PEGylated nanoshells for transdermal and transcranial PACT of a rat brain at $800 \mathrm{~nm}$, with a $10.4 \mathrm{MHz}$ unfocused ultrasonic transducer [64]. The absorption cross section of the nanoshell at $800 \mathrm{~nm}$ is $\sim 1.1 \times 10^{-10}$ $\mathrm{cm}^{2}$, which is $6.4 \times 10^{5}$ times as large as that of hemoglobin at $570 \mathrm{~nm}$.

The scalability of representative PAM systems is summarized in Table 1. By adjusting the system configuration, PAM can be tuned over a wide range of resolution, imaging depth and detection sensitivity, depending on the application.

\section{PAM imaging speed}

As a high-resolution imaging modality, PAM requires 2D raster scanning for 3D imaging. Compared with confocal microscopy and two-photon microscopy, PAM does not need depth scanning. Nonetheless, fast imaging speed is desirable to capture dynamics and eliminate motion artifacts caused by breathing and heart beating.

The traditional PAM systems with ball-screw mechanical scanning have a typical crosssectional (B-scan) scanning rate of $1 \mathrm{~Hz}$ [33-35]. This translates into an image acquisition time of $7 \mathrm{~min}$ for OR-PAM or $1 \mathrm{~min}$ for dark-field AR-PAM over a $1 \times 1 \mathrm{~mm}^{2}$ imaging area. Finer resolution PAM systems, such as SW-PAM, need more pixels per unit area, resulting in an even longer data acquisition time [35].

Recently, great efforts have been invested in improving imaging speed, especially for ORPAM [66-73]. Optical scanning of the excitation laser beam by 2D galvanometer mirrors is used to replace the relatively slow mechanical scanning. As a result, the B-scan rate has been increased up to $1800 \mathrm{~Hz}$ over a scanning range of $\sim 100 \mu \mathrm{m}[66,67]$. Fast in vivo imaging of the microcirculation in mouse skin has been demonstrated, with an $18 \mathrm{~Hz}$ volumetric frame rate [66]. However, unlike optical microscopy, where both the illumination and detection beams can be simultaneously steered, the acoustic beam in PAM cannot be scanned by the same mirrors operating in air. The optical scanning is therefore restricted to the focal spot of the static ultrasonic transducer, resulting in a scanning range of no more than $100 \mu \mathrm{m}$. Besides, the detection sensitivity is not constant within the acoustic focus. To solve the above issues, one method is to use unfocused ultrasonic transducers [68, 
70]. This method relaxes the scanning range to $6 \mathrm{~mm}$, but the acoustic detection sensitivity is reduced by $\sim 40 \mathrm{~dB}$. Another choice is to use cylindrical focusing as a compromise between spherical focusing and unfocusing [74]. Fast optical scanning is performed along the line focus of the transducer, and slow mechanical scanning is performed orthogonally. This hybrid scanning method extends the scanning range to $\sim 4 \mathrm{~mm}$ with a sensitivity loss of $\sim 20 \mathrm{~dB}$.

To increase the imaging speed while maintaining the detection sensitivity, a voice-coil scanning OR-PAM has been developed [71]. By integrating both the optical illumination and acoustic detection on a single-piece scanning head, voice-coil PAM has achieved a Bscan rate of $40 \mathrm{~Hz}$ over a range of $1 \mathrm{~mm}$, without sensitivity loss. However, the scanning speed is largely limited by the mass of the scanning head and the driving force from the voice coil. In addition to OR-PAM, voice-coil scanner has also been used for improving the imaging speed of AR-PAM [75, 76]. Recently, a wide-field fast-scanning OR-PAM enhanced by a water-immersible MEMS (i.e., microelectromechanical system) scanning mirror (MEMS-OR-PAM) has been proposed and implemented [72, 77]. Because it uses an electromagnetic driving force, the whole MEMS scanning system can be submerged in water. By simultaneously scanning the excitation laser beam and resultant acoustic waves, MEMS-OR-PAM maintains confocal alignment and thus high sensitivity over a large field of view. This system has achieved a B-scan rate of $400 \mathrm{~Hz}$ over a $3 \mathrm{~mm}$ range [72].

PAM can also improve its imaging speed through parallel acoustic detection by ultrasound transducer arrays. In a multi-focal OR-PAM system, twenty diffraction-limited focal spots are simultaneously excited, and the resultant PA signals are detected by a 48-element ultrasound linear array [78]. Limited by the 6:1 multiplexing in data acquisition, the imaging speed is eventually improved by three to four times, compared with that in conventional single-focus and single-detector OR-PAM. However, this method needs twentyfold more laser energy than conventional OR-PAM. Besides, because of the inverse reconstruction of PA images, reconstruction artifacts due to the limited detection aperture may degrade the image quality.

Different fast-scanning methods for PAM imaging are summarized in Table 2. Currently, it is the laser repetition rate that limits the imaging speed in PAM. Theoretically, real-time $\left(\geq 30 \mathrm{~Hz}\right.$ ) volumetric imaging over a $1 \times 1 \mathrm{~mm}^{2}$ surface area needs a $5 \mathrm{MHz}$ laser with a pulse energy of $100 \mathrm{~nJ}$ for OR-PAM, or a $50 \mathrm{kHz}$ laser with a pulse energy of $100 \mu \mathrm{J}$ for AR-PAM. To avoid interference between PA waves excited by consecutive pulses, it is the acoustic flight time that limits the ultimate imaging speed. For example, to image a sample with a thickness of $1 \mathrm{~mm}$, the minimum time interval between consecutive pulses should be $0.67 \mu \mathrm{s}$, which corresponds to a maximum laser repetition rate of $1.5 \mathrm{MHz}$.

\section{Multi-contrast PAM}

Although PAM and fluorescence microscopy both start with photon excitation of molecules, PAM can potentially image all molecules. By contrast, fluorescence microscopy can image only a small fraction of molecules, those that exhibit fluorescent relaxation.

\subsection{Endogenous contrast agents}

In biomedical studies, the advantages of endogenous contrast agents are undeniable. They do not induce perturbations to original tissue microenvironment; they are nontoxic and do not require costly and time-consuming regulatory approval. As shown in Figure 4, endogenous absorbers in biological tissue can be classified into two groups, based on their primary absorbing wavelengths. 
In the ultraviolet (UV) $(180 \mathrm{~nm}$ to $400 \mathrm{~nm}$ ) and visible (400 nm to $700 \mathrm{~nm}$ ) regions (UVVIS), the primary absorbers for PA imaging include DNA/RNA [39, 79], cytochrome $c$ [80, 81], myoglobin [80], hemoglobin [82] and melanin [83]. Among them, DNA and RNA absorb mainly in the UV region. By exciting unlabeled DNA and RNA at $266 \mathrm{~nm}$, UV-PAM has achieved noninvasive imaging of cell nuclei in vivo without staining (Figure 5a) [39]. As cancer cells typically have abnormal cell density and nuclear morphology, UV-PAM can potentially be used for histology-free early cancer diagnosis and intraoperative cancer cell detection [84]. Nevertheless, the potential cell damage caused by overexposure to UV light, such as direct and indirect DNA breakage, need to be carefully controlled [85]. If UV-PAM is used to tumor demarcation in the operating room, the exposed tissue can be removed surgically. Cytochrome $c$ in cytoplasm has also been imaged [81]. PAM of both cell nuclei through DNA/RNA absorption and cytoplasm through cytochrome $c$ absorption provides an in vivo label-free equivalent to conventional ex vivo hematoxylin and eosin histology, where hematoxylin stains cell nuclei and eosin labels cytoplasm.

As the most abundant protein in blood and the major oxygen carrier, hemoglobin has been extensively imaged by PAM for a number of hemodynamic parameters, such as total hemoglobin concentration, oxygen saturation, blood flow speed and the metabolic rate of oxygen [82]. In the visible spectral range, the predominant optical absorption of hemoglobin over other absorbers (e.g., water and lipid) enables highly sensitive PAM imaging of microvasculature and even single red blood cells, with an SNR of more than $40 \mathrm{~dB}$ (Figure 5b) [35]. Functional and metabolic PAM imaging of microvasculature assists comprehensive understanding of tumor microenvironment, such as neovascularization, hypoxia and hypermetabolism $[82,86]$. In addition to cancers, PAM has also been used to image microvasculature damage induced by diabetes, another common cause of death in the US [87].

Melanin is the major light absorbing molecule in melanotic melanoma, the foremost killer among skin cancers [88]. Due to its extremely high optical absorption in the UV-VIS region (e.g., $~ 10000$ times that of water and $\sim 100$ times that of oxygenated hemoglobin at $700 \mathrm{~nm}$ ), melanin in melanosomes is an ideal contrast agent for early melanoma detection by PAM $[33,82,83]$. In animals, PAM has been successfully applied to noninvasive in vivo detection of circulating melanoma cells in blood stream and chronic monitoring of melanoma growth, with a penetration depth up to $4 \mathrm{~mm}$ and an SNR of $30 \mathrm{~dB}$ [83, 89-92]. In tissue engineering, spatial distribution of the melanoma cells in the scaffold has also been imaged by PAM [93]. In addition to melanoma detection, melanin also provides good contrast for PAM imaging of the retinal pigment epithelium (RPE) layer in rat eyes with an SNR of 23 $\mathrm{dB}$ (Figure 5c) $[68,94]$. More importantly, the high optical absorption of melanin allows a laser incident fluence of $0.1 \mu \mathrm{J} / \mathrm{cm}^{2}$, which is below the ANSI limit for eye imaging $(0.5 \mu \mathrm{J} /$ $\mathrm{cm}^{2}$ ). Since RPE has critical functions in vision, PAM holds great promise for both fundamental investigation and clinical diagnosis of eye diseases, such as age-related macular degeneration [68, 94].

In the near-infrared region (700 $\mathrm{nm}$ to $1400 \mathrm{~nm}$ ), lipid [35, 95, 96], water [97] and glucose [98] are the major absorbers for PA imaging. Abnormal lipid, water or glucose concentrations usually indicate an unhealthy status. For example, lipid is a common constituent in atherosclerotic plaques, the location and area of which are closely related to the progression of atherosclerosis $[35,95,99]$. PAM can image lipid and water with high resolution in relatively deep tissue, taking advantage of its near-infrared excitation [35, 95, 97]. Blood glucose level is a standard index for diabetes diagnosis and treatment evaluation. Photoacoustic sensing of blood glucose concentration has been demonstrated both in vitro and in humans, with good correlations with the clinical measurements [98]. Compared with the absorbers in the UV-VIS region mentioned above, lipid, water and glucose have been 
less explored by PAM. It is partially because wavelength-tunable high repetition rate lasers typically have low energy in the spectral region beyond $900 \mathrm{~nm}$. OPO or Ti:Sapphire lasers can provide the required optical wavelengths and energy, but they typically have a pulse repetition rate no more than $10 \mathrm{~Hz}$. Nevertheless, with more advanced laser systems, PAM is expected to engage more in imaging near-infrared absorbers.

\subsection{Exogenous contrast agents}

The advantage of exogenous contrast agents over endogenous ones lies in three aspects [8, 19]. First, the chemical and optical properties of exogenous contrast agents can be specifically engineered for different applications. Second, exogenous contrast agents can be conjugated with targeting molecules (e.g., antibodies) to selectively bind to disease-specific cell surface receptors. Third, exogenous contrast agents can be concurrently used for targeting, imaging and therapeutic purposes. So far, organic dyes, nanoparticles, fluorescent proteins and reporter gene products have been used as PAM contrast agents, enabling chemical, molecular and genetic imaging. To achieve a deep penetration depth and avoid background water absorption, most of these exogenous contrasts work in red or nearinfrared (less than $900 \mathrm{~nm}$ ) spectral regions.

Organic dyes have small molecular sizes ( $\leq 3 \mathrm{~nm}$ ) and can be rapidly cleared by glomerular filtration [100]. PEGylated ICG (ICG-PEG) has been used by AR-PAM to enhance cortical vasculature imaging in rats [64]. IRdye 800 has been used for noninvasive brain tumor imaging in mice [65]. Evans blue has been used to enhance capillary imaging in mouse ears by OR-PAM (Figure 5d) [101]. Methylene blue has been used for sentinel lymph node (SLN) imaging for cancer biopsy [102]. 2-NBDG, an optical glucose analog, has been used for brain metabolism study [103]. In general, the PA detection sensitivity of organic dyes is at the micromolar level at $5 \mathrm{~cm}$ depth [64].

Nanoparticles typically have diameters greater than $10 \mathrm{~nm}$, resulting in good optical absorbance but slow clearance from the body [104-111]. Their absorption cross-sections can be orders of magnitude larger than those of organic dye molecules due to plasmon resonance. Their long-circulating feature can be beneficial for cancer drug delivery [100]. So far, a variety of nanoparticles with different sizes, shapes and compositions have been used as PAM contrast agents [8]. Because of their inert chemical properties, gold nanoparticles have been extensively investigated for tumor targeting (Figure 5e) [91, 109, 112, 113], cortical vasculature enhancement [114] and SLN mapping [115-117]. The PA detection sensitivity of nanoparticles is at the picomolar level [64]. Unlike organic dyes, most nanoparticles are still awaiting FDA approval for clinical translation. More studies are still needed to assess their short-term toxicity and long-term effects of accumulation and chronic exposure [118, 119].

The development of genetically encoded fluorescent proteins (FP) has revolutionized molecular cell biology by tracking the dynamics of specific protein. Unlike organic dyes and nanoparticles, FPs are expressed in living cells and do not need complex exogenous delivery. Compared with fluorescence microscopy, PAM can provide deeper tissue imaging of FPs, including eGFP, mCherry and iRFP [120-122]. For example, as shown in Figure 5f, PAMac was used to image a mouse mammary gland tumor expressing iRFP (shown in blue) and its surrounding blood vessels (shown in red) at $4 \mathrm{~mm}$ depth beneath the skin surface [122]. The detection sensitivity of FPs is at the nanomolar level [8].

In addition to FPs, other non-fluorescent reporter gene products can also be imaged by PAM, which makes PAM more flexible than fluorescence imaging. For example, PAM imaging of LacZ gene activity has been demonstrated by using the blue product as the contrast agent, which was produced from LacZ encoded $\beta$-galactosidase and locally injected 
X-gal [123-125]. Given the strong optical absorption of melanin, a few groups have been working on transferring genes to non-melanogenic tumor cells to encode melanin as the contrast agent for PAM imaging [126-128].

\section{Multi-parameter PAM}

Originating from optical absorption, photoacoustic signals can be used to derive a number of physical, chemical and functional parameters of the absorber and its microenvironment. Since a single parameter may not be able to fully reflect the true physiological and pathological conditions, multi-parameter PAM can provide a more comprehensive understanding, thus benefiting the diagnosis, staging and treatment of diseases.

Total concentration of hemoglobin $\left(\mathrm{C}_{\mathrm{Hb}}\right)$ is the most commonly used blood perfusion index. At the isosbestic wavelengths of hemoglobin (e.g., $498 \mathrm{~nm}, 568 \mathrm{~nm}$ and $794 \mathrm{~nm}$ ), the PA signal amplitude reflects the $\mathrm{C}_{\mathrm{Hb}}$ distribution in relative values, regardless of the oxygenation level of hemoglobin (Figure 6a) [82]. Because PA amplitude is also related to the local laser fluence, a phantom calibration or additional correction is needed to obtain the absolute $\mathrm{C}_{\mathrm{Hb}}$.

Oxygen saturation of hemoglobin $\left(\mathrm{sO}_{2}\right)$ is an important indicator of tissue oxygenation and viability. In particular, hypoxia is a hallmark of late stage cancers [129]. A large hypoxic region usually correlates with poor prognosis. From fluence-compensated PAM measurements at two or more wavelengths, the relative concentrations of the two forms of hemoglobin (oxy-hemoglobin $\mathrm{HbO}_{2}$ and deoxy-hemoglobin $\mathrm{HbR}$ ) can be quantified through spectral analysis, and thus $\mathrm{sO}_{2}$ can be computed (Figure 6b) [82, 130-136].

In practice, however, accurate laser fluence compensation for absolute $\mathrm{C}_{\mathrm{Hb}}$ and $\mathrm{sO}_{2}$ quantification can be challenging. Although it is less problematic for OR-PAM because of the superficial targeting depth, the wavelength-dependent light attenuation in deep tissue makes it difficult for AR-PAM to compensate for the local fluence. One solution is to incorporate empirical correction or calibration factors that account for the fluence distribution. For example, the dependence of fluence on optical wavelength can be calibrated by inserting a black plastic absorber beneath the skin of a mouse at the targeted depth [33]. Although useful for small animal models, this method, however, is invasive, thus hard to implement for human studies. Another potential solution is to incorporate PAM with diffuse reflectance spectroscopy [137] or diffuse optical tomography (DOT) [138], which can quantify the tissue optical properties or the fluence distribution. In addition, other model-based inversion methods have also been explored to mathematically reconstruct the tissue optical properties from the detected PA signals, which, however, are typically computationally intensive $[139,140]$. Alternatively, the frequency spectra of PA signals at multiple optical wavelengths can also be used to fit for absolute concentrations of $\mathrm{HbO}_{2}$ and $\mathrm{HbR}$, and thus $\mathrm{C}_{\mathrm{Hb}}$ and $\mathrm{sO}_{2}$, where fluence compensation is not required [141, 142]. In this calibration-free method, multi-wavelength measurements are needed to cancel out the influences of ultrasonic transducer bandwidth and acoustic attenuation.

Blood flow helps keep tissue alive by distributing nutrients, oxygen and other products of metabolism. Using the excellent absorption contrast provided by hemoglobin, PAM can be used to measure blood flow with better contrast to noise ratio than that achieved by backscattering-based methods such as Doppler optical coherence tomography (OCT) and Doppler ultrasound [143-147]. Photoacoustic Doppler flowmetry measures the axial flow speed on the basis of Doppler frequency shift, by using either intensity-modulated continuous-wave (CW) excitation [148, 149] or tone-burst excitation [150]. While the CW method provides high flow measurement accuracy without depth resolution, the tone-burst method trades depth resolution for flow measurement accuracy. Correlation-based 
photoacoustic flowmetry measures the transverse or axial flow speeds by performing temporal autocorrelation [151,152] or cross-correlation [153] over consecutive photoacoustic waveforms, respectively. Taking advantage of its high resolution, OR-PAM has been successfully used to measure blood flow in vivo, with the penetration depth limited to $\sim 1 \mathrm{~mm}$ (Figure 6c) $[61,154,155]$. Although successful in OR-PAM, none of the above methods have been demonstrated for blood flow imaging in AR-PAM yet [156]. Likely, as PAM resolution becomes greater than the spatial separation between individual RBCs or groups of RBCs, blood approximates a spatially homogeneous solution. The signal fluctuations induced by RBC flowing are thus suppressed. Therefore, it remains a challenge to implement the above methods for deep flow imaging [156, 157]. Photoacoustic thermal flowmetry measures the flow speed based on the thermal diffusion, a method that does not rely on the presence of resolvable particles [156]. Similarly, PA imaging of wash-in and wash-out dynamics of nanoparticles or organic dyes can also provide flow information [158-160]. However, these two methods would also have difficulty in deep flow measurement, because they rely on clearly defined illumination boundaries which are difficult to maintain in the optical diffusive regime.

The metabolic rate of oxygen $\left(\mathrm{MRO}_{2}\right)$ directly reflects the oxygen consumption of tissue. Almost all diseases, especially cancer and diabetes, manifest abnormal oxygen metabolism. Hypermetabolism is a hallmark of all kinds of cancers [129, 161]. Currently, by virtue of measuring all parameters required for $\mathrm{MRO}_{2}$ quantification, OR-PAM, as a single modality, is the only imaging technique that can noninvasively quantify absolute $\mathrm{MRO}_{2}$ using endogenous contrast [5, 82]. Moreover, the high resolution of OR-PAM enables the $\mathrm{MRO}_{2}$ studies of the tumor microenvironment. The preliminary results obtained by OR-PAM have demonstrated its early cancer detection capability (Figures 6d-f). This new technology has great potential to advance metabolism-associated studies such as oncology and neurology. Alternatively, PAM can be integrated with Doppler OCT or Doppler ultrasound for $\mathrm{MRO}_{2}$ quantification, where PAM is used for blood oxygenation measurement while Doppler OCT or Doppler ultrasound quantifies blood flow $[162,163]$.

In addition to measuring the electronic absorption of molecules, PAM is also capable of measuring their overtone vibrational absorption in the near-infrared spectral region, which can reveal the selective tissue composition and its molecular structure, such as lipid in artery walls. PAM has been used to image the second overtone of the $\mathrm{CH}$ bond stretch of fat bodies in a 3rd-instar larva of Drosophila melanogaster in vivo at $1200 \mathrm{~nm}$, demonstrating its chemically-selective imaging capability (Figure 6g) [95]. PAM can also be combined with stimulated Raman excitation for improved chemical specificity [96]. However, vibrational PAM and stimulated Raman PAM may both suffer from lower signal strength compared with traditional PAM, because the overtone transitions and Raman shift are orders of magnitude weaker than electronic transitions. To some extent, this drawback is overcome by excitation in the near-infrared region, where ANSI allows higher incident laser intensity. In addition, PAM has been used for imaging Förster resonance energy transfer (FRET), the efficiency of which reflects intra- and inter-molecular distances in the 1 to $10 \mathrm{~nm}$ range [164]. Compared with fluorescence FRET imaging, PA FRET imaging is expected to apply in deeper tissue.

Absorption relaxation time is an important material property that describes the average time that an excited molecule spends in the excited state before returning to its ground state.

Since absorption relaxation time is typically at the picosecond level, it is usually measured by using an expensive femtosecond or picosecond laser. By contrast, by fitting the saturation curve of the signal amplitude as a function of incident laser intensity, PAM is able to measure the picosecond absorption relaxation times of natural materials, such as $\mathrm{HbO}_{2}$ and $\mathrm{HbR}$, in reflection-mode with a nanosecond laser (Figure 6h) $[60,165]$. Based on the 
relaxation time contrast, $\mathrm{PAM}$ can even measure $\mathrm{sO}_{2}$ in a mouse ear in vivo using a single wavelength at $576 \mathrm{~nm}$ (Figure 6i) [165]. Compared with the traditional two-wavelength $\mathrm{sO}_{2}$ measurement, on one hand, this relaxation time based method is more cost-effective because single wavelength lasers are typically much more compact and less costly than wavelengthtunable lasers. On the other hand, to better fit the saturation curve, more than two measurements with different incident intensities are typically required, which prolongs the image acquisition time [165]. Besides, to observe saturation requires much stronger incident intensities, which may exceed the ANSI limit [165]. This, however, can be overcome by shortening the pulse width.

Two other material parameters, dichroism [166] and magnetomotion [167, 168], can also be used by PAM to enhance imaging specificity. In dichroism PAM, the distinct absorption responses of dichroic molecules (e.g., amyloid plaques and retinal nerve fibers) to differently polarized optical irradiations enable differential detection, which can eliminate the non-dichroic background and greatly enhance specificity. As a demonstration, the linear dichroism of Congo Red labeled amyloid plaques was imaged ex vivo (Figures 7a-b) [166]. In magnetomotive PAM, the displacement of magnetic nanoparticles (MNPs) under the manipulation of an external magnetic field enables motion detection, which can suppress the non-magnetomotive background and thus enhance the specificity remarkably (Figures 7cd). Notwithstanding, both dichroism PAM and magnetomotive PAM are applicable to only a limited number of targets. Besides, with increasing imaging depth, dichroism PAM may also suffer from optical depolarization by tissue [169].

In addition to absorber properties, PAM can also measure the microenvironment properties, including $\mathrm{pH}$ and partial oxygen pressure $\left(\mathrm{pO}_{2}\right)[170,171]$. A pH-sensitive fluorescent dye (SNARF-5F carboxylic acid) was used as the contrast agent for PAM imaging of $\mathrm{pH}$ in tissue phantoms at depths up to $2 \mathrm{~mm}$ [171]. The $\mathrm{pH}$ was computed from the relative concentrations of acidic and basic populations of SNARF-5F molecules, which were spectrally unmixed using a two-wavelength measurement at $581 \mathrm{~nm}$ and $594 \mathrm{~nm}$, like that for $\mathrm{sO}_{2}$ quantification. Physiologically realistic $\mathrm{pH}$ values from 6.78 to 7.80 were measured by PAM with an error less than $2 \%$. The challenge ahead of $\mathrm{pH}$ measurement in vivo is to compete with the strong absorption of blood. Besides, the accuracy of $\mathrm{pH}$ measurement is also affected by the wavelength-dependent light attenuation by tissue.

An oxygen-sensitive dye Pd-tetra-(4-carboxyphenyl)tetrabenzoporphyrin dendrimer (G2) has been used as the contrast agent for PAM measurement of $\mathrm{pO}_{2}$ [170]. The phosphorescent lifetime of the $\mathrm{G} 2$ dye is shortened in the presence of oxygen. A pumpprobe method has been used to measure the G2 lifetime. PA signal amplitude induced by the probe beam reflects the remaining population of excited G2 molecules at the intermediate triplet state. By varying the delay time between the probe beam $(950 \mathrm{~nm})$ and pump beam $(630 \mathrm{~nm})$, an exponential decay curve of PA amplitude can be obtained, from which the dye lifetime can be fitted for. Compared with the spectroscopic $\mathrm{sO}_{2}$ measurement, the lifetime based $\mathrm{pO}_{2}$ measurement does not rely on the absolute $\mathrm{PA}$ amplitude, thus the measurement accuracy is not affected by heterogeneous optical properties and inhomogeneous light fluence in the background. Nevertheless, this method may suffer from weak PA signal strength due to the low fluorescence quantum yield ( 10\%) of the dye. In addition to G2 dye, other oxygen-sensitive dyes such as Pt(II) octaethylporphine and methylene blue were also explored by PAM for oxygen sensing [172, 173].

The different parameters measured by PAM are summarized in Table 3. 


\section{PAM applications in biomedicine}

By offering high-resolution images with unique optical contrast, PAM has so far been applied to numerous preclinical and human studies, including vascular biology [174-176], oncology [65, 83, 124, 151, 177-180], neurology [64, 132, 181, 182], ophthalmology [68, $70,94,135,183]$, dermatology [184-188], gastroenterology [36, 57, 189-191], and cardiology $[35,99,192,193]$. In the interest of brevity, only a few representative applications are highlighted in this paper. Information about additional applications can be found in recent Reviews [8, 20, 21, 157].

Microcirculation provides exchange sites for gases, nutrients, metabolic wastes, and thermal energy between blood and tissue. The high spatial resolution of PAM enables the microcirculation studies of small animal models, and provides insight into disease-induced abnormal microcirculations in humans. AR-PAM has been used to study the hemodynamic changes induced by electrical stimulations in the mouse brain [181]. OR-PAM was used to study the microvascular vasodilation and vasomotion in a mouse ear in response to oxygenation challenges [133] (Figures 8a-b), the $\mathrm{sO}_{2}$ mapping at the capillary level in the mouse brain, eye and small intestine [132, 135, 191], the Evans blue dye-enhanced capillary network imaging in the mouse ear [101], blood pulse wave propagation measurement [194] and in vivo zebrafish larva imaging [195].

Chronic imaging of the same animal both eliminates inter-animal heterogeneity, and allows direct visualization of long-term physiological and pathological progression (e.g., aging and carcinogenesis). The noninvasiveness of PAM is particularly well suited for chronic imaging of small animal models, using endogenous contrast agents (e.g., melanin and hemoglobin).

For example, AR-PAM has been used to continuously monitor the growth of a melanoma in a mouse brain for two weeks [83]. OR-PAM has been used for serial imaging of neovascularization in the same mouse for two months (Figures 8c) [174, 175]. OR-PAM has also been used to monitor the healing process of laser-induced microvascular lesions in a mouse ear for over 12 days [134]. The chronic imaging capability of PAM enables minimal animal preparation and reduces the number of animals required for statistical study.

In living organisms, biological events happen at time scales as short as milliseconds. PAM, a fast imaging modality, has been applied to various dynamic studies. For example, a lineararray-based real-time PAM has been used to studies of cardiovascular dynamics of a mouse heart [193], blood pulsatile dynamics in a human forearm [188], and uptake and clearance dynamics of Evans blue dye in SLNs of a mouse [196]. Furthermore, as shown in Figure 8d, the wash-in dynamics of Evans blue dye in the microcirculation of a mouse ear were imaged in vivo at a B-scan frame rate of $250 \mathrm{~Hz}$ and a C-scan frame rate of $0.5 \mathrm{~Hz}$ [160]. Such a temporal resolution allows PAM to capture the whole dynamic flow of contrast agent from arteries to veins.

In noisy tissue microenvironment especially in the brain, high detection sensitivity is essential for studying weak physiological changes in response to either internal signaling or external stimulation. PAM provides inherently background-free detection with $100 \%$ sensitivity. AR-PAM has been used to study the temporal variations in a mouse brain in response to oxygenation alternating between hyperoxia and hypoxia [197] (Figures 9a-b). A detection limit of $3.6 \%$ change in oxygenation has been reported, which enables PAM monitoring of weak brain activity via hemodynamic responses. AR-PAM has also been used to image ICG enhanced single walled nanoparticles (ICG-SWNP) with a detection sensitivity of $170 \mathrm{pM}$ [178]. When ICG-SWNPs are targeted to tumor cell surfaces, ARPAM can detect as few as $\sim 10^{4}$ cancer cells. Such a high detection sensitivity is needed for 
early cancer diagnosis, because the malignant switching in cancer progression typically needs $\sim 10^{5}$ cells [100].

For clinical applications, PAM must follow the safety limits to avoid potential damage to patients. PA signal amplitude is typically less than $10 \mathrm{kPa}$, orders of magnitude lower than that of diagnostic clinical ultrasound scanners. Therefore, hazards due to ultrasound exposure are not of concern. The safety considerations about PA imaging are mostly related to the laser exposure. ANSI has detailed standards for laser exposure to the skin and eye (Laser Institute of America 2007). For example, in the visible spectral range (400 nm to 700 $\mathrm{nm})$, the maximum permissible exposure (MPE) values for the skin are $20 \mathrm{~mJ} / \mathrm{cm}^{2}$ for a single pulse and $200 \mathrm{~mW} / \mathrm{cm}^{2}$ for the average power. Of course, the safety standards are lower than the damage thresholds. Therefore, it might be justifiable to use laser exposure above the ANSI limits but below the damage thresholds in some applications. Fortunately, due to the adequate conversion efficiency from temperature to acoustic pressure (i.e., $1 \mathrm{mK}$ temperature rise leads to $\sim 800 \mathrm{~Pa}$ pressure rise [2]), PAM is safe for skin and eye imaging, which clears a major hurdle for its clinical translation. Imaging of the subcutaneous microvasculature of a human palm in vivo has been demonstrated by AR-PAM at $584 \mathrm{~nm}$, with an incident fluence of $\sim 6 \mathrm{~mJ} / \mathrm{cm}^{2}$ and penetration depth of $3 \mathrm{~mm}$ (Figure 9c) [33]. The average contrast to noise ratio is $51 \mathrm{~dB}$. OR-PAM has been used to image the ocular microvasculature of a living mouse at $570 \mathrm{~nm}$, with an incident fluence of $\sim 0.1 \mu \mathrm{J} / \mathrm{cm}^{2}$, which is below the MPE for eye imaging (Figure 9d) [135]. Individual RBCs travelling in iris capillaries can be detected with a contrast to noise ratio of $\sim 20 \mathrm{~dB}$.

\section{Summary and discussions}

In summary, we have reviewed the characteristics and applications of different PAM implementations. PAM has the following notable features: (1) PAM breaks through the optical diffusion limit, with highly scalable spatial resolution and maximum imaging depth in both the optical and acoustic domains. (2) PAM images optical absorption contrast with $100 \%$ sensitivity, and provides images without speckle artifacts. (3) PAM can essentially image all molecules at their absorbing wavelengths. (4) PAM is capable of functional and metabolic imaging in absolute units using endogenous contrast agents.

As in modern optical microscopy, it is possible to combine multiple optical objectives with different magnification powers to a single OR-PAM system for fast resolution switching. However, the confocal alignment of the optical illumination and acoustic detection needs to be maintained. Typically, a microscope objective with a higher NA comes with a shorter focal length. To keep the imaged object in focus, one solution is to use a specially designed parfocal lens, which is capable of varying the effective NA without changing the optical focal length. Similarly, it is also possible to integrate multiple ultrasonic transducers with different central frequencies into a single AR-PAM system, while keeping the same illumination. Furthermore, OR-PAM can be combined with AR-PAM as a single device to cover a wider scale range. Since OR-PAM and AR-PAM can share the same detection, only the optical illumination needs to be integrated.

Integration between PAM and other imaging modalities can provide complementary contrasts, thus is a direction for future PAM development. So far, PAM has been integrated with back-scattered optical microscopy (scattering contrast) [198], fluorescence microscopy (fluorescence contrast) [70, 199, 200], optical coherence tomography (scattering contrast) [70, 201], two-photon microscopy (fluorescence contrast) [202] and pulse-echo ultrasound imaging (mechanical and elastic contrast) [203, 204]. In these integrated systems, the two sub-systems share either the same illumination or detection. Therefore, their images are inherently co-registered. In addition to providing complementary contrast, PAM has been 
combined with a high intensity focused ultrasound (HIFU) system for therapeutics, where PAM is used to outline the location and shape of tumors and guide the subsequent HIFU therapy or drug release $[177,205]$. In addition to the integration of instrumentations, new exogenous contrast agents have also been integrated for multi-modal systems. For example, perfluorocarbon nanoparticles loaded with near-infrared dyes have been used as a dual contrast agent for fluorescence imaging and PAM imaging [206].

Another direction is to miniaturize the PAM system for internal organ and intravascular imaging [99, 190, 195, 207-211]. The first sideway looking photoacoustic endoscope (PAE) with a 4.2-mm-diameter probe has been demonstrated in acoustic-resolution mode for functional esophagus and colon imaging on small animal models [190, 207]. The bottleneck for further miniaturization of the PAE probe involves integrating the light delivery fiber with a conventional piezoelectric transducer. Despite the complex fabrication procedures for miniature piezoelectric detector, its detection sensitivity decreases with its size. By contrast, optical detection of ultrasound pressure using a transparent Fabry-Perot sensor may be an alternative solution [212]. Theoretically, PAE should enjoy the same scalability as PAM. Since most cancers start from the epithelial layer, PAE can be implemented in opticalresolution mode for high-resolution epithelial imaging of early-stage cancer [190, 195, 207]. Delivery of coupling medium for acoustic propagation remains another challenge for PAE imaging of internal lumens such as the trachea and gastrointestinal (GI) tract. It has been suggested that water and ultrasound gel can be introduced to the GI tract [190, 195]. However, it is challenging to apply water to the respiratory system. An inflatable balloon with a water filling channel could be a solution [213]. By contrast, it is less problematic for intravascular PAE imaging since blood is a natural acoustic coupling medium. However, the strong absorption of blood in the visible spectral range requires the use of near-infrared wavelengths. Alternatively, blood can be flushed with saline.

As a maturing imaging technique, PAM is expected to find new applications in both fundamental life science and clinical practice, which include but are not limited to tumor angiogenesis, lymphatic dynamics, neural activity, brain metabolism, cancer detection, drug delivery and intraoperative monitoring. While PAM has been commercialized for preclinical applications, future commercialization for clinical applications will greatly accelerate the translation of PAM from a lab technology to a mainstream imaging modality.

\section{Acknowledgments}

The authors appreciate Prof. James Ballard's close reading of the manuscript and useful discussions from Konstantin Maslov, Lidai Wang, Chi Zhang, Dakang Yao, Amos Danielli, Arie Krumholz and Amy Winkler. This work was sponsored by National Institutes of Health (NIH) grants R01 EB000712, R01 EB008085, R01 CA134539, U54 CA136398, R01 CA157277, and R01 CA159959. L. Wang has a financial interest in Endra, Inc., and Microphotoacoustics, Inc., which, however, did not support this work.

\section{References}

1. Amos B. Lessons from the history of light microscopy. Nature Cell Biology. 2000; 2:E151-E152.

2. Wang, LV.; Wu, H.-i. Biomedical optics : principles and imaging. Vol. xiv. Wiley-Interscience; Hoboken, N.J.: 2007. p. 362

3. Wang LHV, Hu S. Photoacoustic Tomography: In Vivo Imaging from Organelles to Organs. Science. 2012; 335:1458-1462. [PubMed: 22442475]

4. Wang LV. Tutorial on photoacoustic microscopy and computed tomography. IEEE Journal of Selected Topics in Quantum Electronics. 2008; 14:171-179.

5. Wang LV. Prospects of photoacoustic tomography. Medical Physics. 2008; 35:5758-5767. [PubMed: 19175133] 
6. Wang LV. Multiscale photoacoustic microscopy and computed tomography. Nature Photonics. 2009; 3:503-509. [PubMed: 20161535]

7. Yao J, Wang LV. Photoacoustic tomography: fundamentals, advances and prospects. Contrast Media Mol Imaging. 2011; 6:332-345. [PubMed: 22025335]

8. Kim C, Favazza C, Wang LHV. In Vivo Photoacoustic Tomography of Chemicals: High-Resolution Functional and Molecular Optical Imaging at New Depths. Chemical Reviews. 2010; 110:27562782. [PubMed: 20210338]

9. Li CH, Wang LHV. Photoacoustic tomography and sensing in biomedicine. Physics in Medicine and Biology. 2009; 54:R59-R97. [PubMed: 19724102]

10. Oraevsky AA, Jacques SL, Tittel FK. Measurement of tissue optical properties by time-resolved detection of laser-induced transient stress. Applied Optics. 1997; 36:402-415. [PubMed: 18250688]

11. Esenaliev RO, Karabutov AA, Oraevsky AA. Sensitivity of laser optoacoustic imaging in detection of small deeply embedded tumors. IEEE Journal of Selected Topics in Quantum Electronics. 1999; 5:981-988.

12. Kruger RA, et al. Photoacoustic Ultrasound (Paus) - Reconstruction Tomography. Medical Physics. 1995; 22:1605-1609. [PubMed: 8551984]

13. Kruger RA, Reinecke DR, Kruger GA. Thermoacoustic computed tomography-technical considerations. Medical Physics. 1999; 26:1832-1837. [PubMed: 10505871]

14. Viator JA, Jacques SL, Prahl SA. Depth profiling of absorbing soft materials using photoacoustic methods. IEEE Journal of Selected Topics in Quantum Electronics. 1999; 5:989-996.

15. Calasso IG, Craig W, Diebold GJ. Photoacoustic point source. Physical Review Letters. 2001; 86:3550-3553. [PubMed: 11328020]

16. Grashin PS, et al. Distribution of the laser radiation intensity in turbid media: Monte Carlo simulations, theoretical analysis, and results of optoacoustic measurements. Quantum Electronics. 2002; 32:868-874.

17. Andreev VG, Karabutov AA, Oraevsky AA. Detection of ultrawide-band ultrasound pulses in optoacoustic tomography. Ieee Transactions on Ultrasonics Ferroelectrics and Frequency Control. 2003; 50:1383-1390.

18. Sehgal CM, Greenleaf JF. Scattering of Ultrasound by Tissues. Ultrasonic Imaging. 1984; 6:60-80. [PubMed: 6540912]

19. Luke GP, Yeager D, Emelianov SY. Biomedical applications of photoacoustic imaging with exogenous contrast agents. Annals of biomedical engineering. 2012; 40:422-437. [PubMed: 22048668]

20. Hu S, Wang LV. Neurovascular photoacoustic tomography. Front Neuroenergetics. 2010; 2:10. [PubMed: 20616885]

21. Hu S, Wang LV. Photoacoustic imaging and characterization of the microvasculature. Journal of Biomedical Optics. 2010; 15:011101. [PubMed: 20210427]

22. Emelianov SY, Li PC, O'donnell M. Photoacoustics for molecular imaging and therapy. Physics Today. 2009; 62:34-39. [PubMed: 20523758]

23. Karabutov AA, Savateeva EV, Oraevsky AA. Optoacoustic tomography: New modality of laser diagnostic systems. Laser Physics. 2003; 13:711-723.

24. Brecht HP, et al. Whole-body three-dimensional optoacoustic tomography system for small animals. Journal of Biomedical Optics. 2009; 14:064007. [PubMed: 20059245]

25. Ermilov SA, et al. Laser optoacoustic imaging system for detection of breast cancer. Journal of Biomedical Optics. 2009; 14:024007. [PubMed: 19405737]

26. Wang XD, et al. Noninvasive laser-induced photoacoustic tomography for structural and functional in vivo imaging of the brain. Nature Biotechnology. 2003; 21:803-806.

27. Xu MH, Wang LHV. Universal back-projection algorithm for photoacoustic computed tomography. Physical Review E. 2005; 71:016706.

28. Kruger RA, et al. Photoacoustic angiography of the breast. Medical Physics. 2010; 37:6096-6100. [PubMed: 21158321] 
29. Burgholzer P, et al. Exact and approximative imaging methods for photoacoustic tomography using an arbitrary detection surface. Physical Review E. 2007; 75:046706.

30. Manohar S, et al. The Twente Photoacoustic Mammoscope: system overview and performance. Physics in Medicine and Biology. 2005; 50:2543-2557. [PubMed: 15901953]

31. Gamelin J, et al. Curved array photoacoustic tomographic system for small animal imaging. Journal of Biomedical Optics. 2008; 13:024007. [PubMed: 18465970]

32. Maslov K, et al. Optical-resolution photoacoustic microscopy for in vivo imaging of single capillaries. Optics Letters. 2008; 33:929-931. [PubMed: 18451942]

33. Zhang HF, et al. Functional photoacoustic microscopy for high-resolution and noninvasive in vivo imaging. Nature Biotechnology. 2006; 24:848-851.

34. Maslov K, Stoica G, Wang LHV. In vivo dark-field reflection-mode photoacoustic microscopy. Optics Letters. 2005; 30:625-627. [PubMed: 15791997]

35. Zhang C, Maslov K, Wang LHV. Subwavelength-resolution label-free photoacoustic microscopy of optical absorption in vivo. Optics Letters. 2010; 35:3195-3197. [PubMed: 20890331]

36. Yao J, et al. Double-illumination photoacoustic microscopy. Optics Letters. 2012; 37:659-661. [PubMed: 22344139]

37. Zhang C, et al. Reflection-mode submicron-resolution in vivo photoacoustic microscopy. Journal of Biomedical Optics. 2012; 17:020501. [PubMed: 22463018]

38. Hu S, Maslov K, Wang LV. Second-generation optical-resolution photoacoustic microscopy with improved sensitivity and speed. Optics Letters. 2011; 36:1134-1136. [PubMed: 21479007]

39. Yao D, et al. In vivo label-free photoacoustic microscopy of cell nuclei by excitation of DNA and RNA. Opt. Lett. 2010; 35:4139-4141. [PubMed: 21165116]

40. Kolkman RGM, et al. In vivo photoacoustic imaging of blood vessels using an extreme-narrow aperture sensor. IEEE Journal of Selected Topics in Quantum Electronics. 2003; 9:343-346.

41. Maslov K, Wang LV. Photoacoustic imaging of biological tissue with intensity-modulated continuous-wave laser. Journal of Biomedical Optics. 2008; 13

42. Azhari, H. Basics of biomedical ultrasound for engineers. IEEE; Wiley: Hoboken, N.J: 2010.

43. Stein, EW.; Maslov, K.; Wang, LV. Noninvasive mapping of the electrically stimulated mouse brain using photoacoustic microscopy. SPIE; San Jose, CA, USA: 2008.

44. Wells PNT. Ultrasonic imaging of the human body. Reports on Progress in Physics. 1999; 62:671722.

45. Song KH, Wang LV. Deep reflection-mode photoacoustic imaging of biological tissue. Journal of Biomedical Optics. 2007; 12:060503. [PubMed: 18163798]

46. Song KH, Wang LV. Noninvasive photoacoustic imaging of the thoracic cavity and the kidney in small and large animals. Medical Physics. 2008; 35:4524-4529. [PubMed: 18975699]

47. Song KH, et al. Noninvasive photoacoustic identification of sentinel lymph nodes containing methylene blue in vivo in a rat model. Journal of Biomedical Optics. 2008; 13:054033-6. [PubMed: 19021413]

48. Li ML, et al. Improved in vivo photoacoustic microscopy based on a virtual-detector concept. Optics Letters. 2006; 31:474-476. [PubMed: 16496891]

49. Liao CK, Li ML, Li PC. Optoacoustic imaging with synthetic aperture focusing and coherence weighting. Optics Letters. 2004; 29:2506-2508. [PubMed: 15584276]

50. Passler K, et al. Scanning acoustic-photoacoustic microscopy using axicon transducers. Biomedical Optics Express. 2010; 1:318-323. [PubMed: 21258469]

51. Durnin J, Miceli JJ, Eberly JH. Comparison of Bessel and Gaussian Beams. Optics Letters. 1988; 13:79-80. [PubMed: 19741986]

52. Chen SL, et al. Polymer Microring Resonators for High-Sensitivity and Wideband Photoacoustic Imaging. IEEE Transactions on Ultrasonics Ferroelectrics and Frequency Control. 2009; 56:24822491.

53. Xie ZX, et al. Pure optical photoacoustic microscopy. Optics Express. 2011; 19:9027-9034.

[PubMed: 21643156] 
54. Zhang EZ, et al. Multimodal photoacoustic and optical coherence tomography scanner using an all optical detection scheme for 3D morphological skin imaging. Biomedical Optics Express. 2011; 2:2202-2215. [PubMed: 21833358]

55. Zhang, EZ., et al. Multimodal simultaneous photoacoustic tomography, optical resolution microscopy, and OCT system. SPIE; San Francisco, California, USA: 2010.

56. Matcher SJ, Cope M, Delpy DT. In vivo measurements of the wavelength dependence of tissuescattering coefficients between 760 and $900 \mathrm{~nm}$ measured with time-resolved spectroscopy. Applied Optics. 1997; 36:386-396. [PubMed: 18250686]

57. Yao, J., et al. Double-illumination photoacoustic microscopy of intestinal hemodynamics following massive small bowel resection. SPIE; San Francisco, California, USA: 2012.

58. Bost W, et al. High frequency optoacoustic microscopy. Conf Proc IEEE Eng Med Biol Soc. 2009; 2009:5883-6. [PubMed: 19964880]

59. Ku G, Wang LV. Deeply penetrating photoacoustic tomography in biological tissues enhanced with an optical contrast agent. Optics Letters. 2005; 30:507-509. [PubMed: 15789718]

60. Danielli A, et al. Picosecond absorption relaxation measured with nanosecond laser photoacoustics. Applied Physics Letters. 2010; 97:163701. [PubMed: 21079726]

61. Yao J, et al. In vivo photoacoustic imaging of transverse blood flow by using Doppler broadening of bandwidth. Optics Letters. 2010; 35:1419-1221. [PubMed: 20436589]

62. Laser Institute of America. American National Standard for Safe Use of Lasers Standard. Z136:12007.

63. Wang YW, et al. Photoacoustic tomography of a nanoshell contrast agent in the in vivo rat brain. Nano Letters. 2004; 4:1689-1692.

64. Wang XD, et al. Noninvasive photoacoustic angiography of animal brains in vivo with nearinfrared light and an optical contrast agent. Optics Letters. 2004; 29:730-732. [PubMed: 15072373]

65. Li M, et al. Simultaneous Molecular and Hypoxia Imaging of Brain Tumors In Vivo Using Spectroscopic Photoacoustic Tomography. Proceedings of the IEEE. 2008; 96:481-489.

66. Rao B, et al. Real-time four-dimensional optical-resolution photoacoustic microscopy with Au nanoparticle-assisted subdiffraction-limit resolution. Optics Letters. 2011; 36:1137-1139. [PubMed: 21479008]

67. Hajireza P, Shi W, Zemp RJ. Label-free in vivo fiber-based optical-resolution photoacoustic microscopy. Optics Letters. 2011; 36:4107-4109. [PubMed: 22002401]

68. Jiao SL, et al. Photoacoustic ophthalmoscopy for in vivo retinal imaging. Optics Express. 2010; 18:3967-3972. [PubMed: 20389409]

69. Jiao SL, et al. Simultaneous multimodal imaging with integrated photoacoustic microscopy and optical coherence tomography. Optics Letters. 2009; 34:2961-2963. [PubMed: 19794782]

70. Xie ZX, et al. Laser-scanning optical-resolution photoacoustic microscopy. Optics Letters. 2009; 34:1771-1773. [PubMed: 19529698]

71. Wang LD, et al. Fast voice-coil scanning optical-resolution photoacoustic microscopy. Optics Letters. 2011; 36:139-141. [PubMed: 21263479]

72. Yao J, et al. Water-Immersible MEMS Scanning Mirror Enhanced Optical-Resolution Photoacoustic Microscopy. Biomedical Optics: Optical Society of America. 2012

73. Harrison T, et al. Combined photoacoustic and ultrasound biomicroscopy. Optics Express. 2009; 17:22041-22046. [PubMed: 19997449]

74. Rao B, et al. Hybrid-scanning optical-resolution photoacoustic microscopy for in vivo vasculature imaging. Optics Letters. 2010; 35:1521-1523. [PubMed: 20479795]

75. Wang L, et al. Video-rate functional photoacoustic microscopy at depths. Journal of Biomedical Optics. 2012; 17:106007. [PubMed: 23224006]

76. Harrison T, et al. Combined photoacoustic and ultrasound biomicroscopy. Optics Express. 2009; 17:22041-22046. [PubMed: 19997449]

77. Yao J, et al. Wide-field fast-scanning photoacoustic microscopy based on a water-immersible MEMS scanning mirror. Journal of Biomedical Optics. 2012; 17:080505. [PubMed: 23224156] 
78. Song L, Maslov K, Wang LV. Multifocal optical-resolution photoacoustic microscopy in vivo. Optics Letters. 2011; 36:1236-1238. [PubMed: 21479041]

79. Yao DK, et al. Optimal ultraviolet wavelength for in vivo photoacoustic imaging of cell nuclei. Journal of Biomedical Optics. 2012; 17:056004. [PubMed: 22612127]

80. Zhang C, et al. Label-free photoacoustic microscopy of myocardial sheet architecture. Journal of Biomedical Optics. 2012; 17:060506. [PubMed: 22734729]

81. Zhang C, et al. Label-free photoacoustic microscopy of cytochrome c in cells. Journal of Biomedical Optics. submitted.

82. Yao J, et al. Label-free oxygen-metabolic photoacoustic microscopy in vivo. Journal of Biomedical Optics. 2011; 16:076003. [PubMed: 21806264]

83. Staley J, et al. Growth of melanoma brain tumors monitored by photoacoustic microscopy. Journal of Biomedical Optics. 2010; 15:040510. [PubMed: 20799777]

84. Zink D, Fischer AH, Nickerson JA. Nuclear structure in cancer cells. Nature Reviews Cancer. 2004; 4:677-687.

85. Sinha RP, Hader DP. UV-induced DNA damage and repair: a review. Photochemical \& Photobiological. Sciences. 2002; 1:225-236.

86. Hu, S., et al. Multi-contrast photoacoustic microscopy. SPIE; San Francisco, California, USA: 2012.

87. Krumholz A, et al. Functional photoacoustic microscopy of diabetic vasculature. Journal of Biomedical Optics. 2012; 17:060502. [PubMed: 22734725]

88. American Cancer Society. Cancer facts \& figures 2008. American Cancer Society; Atlanta, GA: 2008.

89. Galanzha EI, et al. In vivo, Noninvasive, Label-Free Detection and Eradication of Circulating Metastatic Melanoma Cells Using Two-Color Photoacoustic Flow Cytometry with a Diode Laser. Cancer Research. 2009; 69:7926-7934. [PubMed: 19826056]

90. Weight RM, et al. Photoacoustic detection of metastatic melanoma cells in the human circulatory system. Optics Letters. 2006; 31:2998-3000. [PubMed: 17001379]

91. Galanzha EI, et al. In vivo magnetic enrichment and multiplex photoacoustic detection of circulating tumour cells. Nature Nanotechnology. 2009; 4:855-860.

92. Wang Y, et al. Fiber-laser-based photoacoustic microscopy and melanoma cell detection. Journal of Biomedical Optics. 2011; 16:011014. [PubMed: 21280901]

93. Zhang Y, et al. Chronic label-free volumetric photoacoustic microscopy of melanoma cells in three-dimensional porous Scaffolds. Biomaterials. 2010; 31:8651-8658. [PubMed: 20727581]

94. Song W, et al. Integrating photoacoustic ophthalmoscopy with scanning laser ophthalmoscopy, optical coherence tomography, and fluorescein angiography for a multimodal retinal imaging platform. Journal of Biomedical Optics. 2012; 17:061206-7. [PubMed: 22734736]

95. Wang HW, et al. Label-Free Bond-Selective Imaging by Listening to Vibrationally Excited Molecules. Physical Review Letters. 2011; 106:238106. [PubMed: 21770549]

96. Yakovlev VV, et al. Stimulated Raman photoacoustic imaging. Proceedings of the National Academy of Sciences of the United States of America. 2010; 107:20335-20339. [PubMed: 21059930]

97. Xu Z, Li CH, Wang LV. Photoacoustic tomography of water in phantoms and tissue. Journal of Biomedical Optics. 2010; 15:036019. [PubMed: 20615021]

98. MacKenzie HA, et al. Advances in photoacoustic noninvasive glucose testing (vol 45, pg 1587, 1999). Clinical Chemistry. 1999; 45:1587-1595. [PubMed: 10471673]

99. Wang B, et al. Intravascular Photoacoustic Imaging. IEEE Journal of Selected Topics in Quantum Electronics. 2010; 16:588-599.

100. Frangioni JV. New technologies for human cancer imaging. Journal of Clinical Oncology. 2008; 26:4012-4021. [PubMed: 18711192]

101. Yao JJ, et al. Evans blue dye-enhanced capillary-resolution photoacoustic microscopy in vivo. Journal of Biomedical Optics. 2009; 14:054049. [PubMed: 19895150]

102. Erpelding TN, et al. Sentinel lymph nodes in the rat: noninvasive photoacoustic and US imaging with a clinical US system. Radiology. 2010; 256:102-110. [PubMed: 20574088] 
103. Yao J, et al. Noninvasive photoacoustic computed tomography of mouse brain metabolism in vivo. Neuroimage. 2012; 64C:257-266. [PubMed: 22940116]

104. Bost W, et al. High resolution optoacoustic detection of nanoparticles on living cells. Ultrasonics Symposium (IUS), 2009 IEEE International. 2009

105. Brannon-Peppas L, Blanchette JO. Nanoparticle and targeted systems for cancer therapy. Adv Drug Deliv Rev. 2004; 56:1649-1659. [PubMed: 15350294]

106. Bulte, JWM. Fundamental biomedical technologies. Vol. Vol. 3. Springer; New York, NY. Berlin Heidelberg: 2007. Nanoparticles in biomedical imaging emerging technologies and applications. Online-Ressource

107. Cho EC, et al. Measuring the Optical Absorption Cross Sections of Au-Ag Nanocages and Au Nanorods by Photoacoustic Imaging. Journal of Physical Chemistry C. 2009; 113:9023-9028.

108. Yang XM, et al. Nanoparticles for photoacoustic imaging. Wiley Interdisciplinary ReviewsNanomedicine and Nanobiotechnology. 2009; 1:360-368. [PubMed: 20049803]

109. Copland JA, et al. Bioconjugated gold nanoparticles as a molecular based contrast agent: Implications for imaging of deep tumors using optoacoustic tomography. Molecular Imaging and Biology. 2004; 6:341-349. [PubMed: 15380744]

110. Kim JW, et al. Golden carbon nanotubes as multimodal photoacoustic and photothermal highcontrast molecular agents. Nature Nanotechnology. 2009; 4:688-694.

111. Cai X, et al. Multiscale Photoacoustic Microscopy of Single-Walled Carbon NanotubeIncorporated Tissue Engineering Scaffolds. Tissue Engineering Part C-Methods. 2012; 18:310317. [PubMed: 22082018]

112. Kim C, et al. In Vivo Molecular Photoacoustic Tomography of Melanomas Targeted by Bioconjugated Gold Nanocages. Acs Nano. 2010; 4:4559-4564. [PubMed: 20731439]

113. Eghtedari M, et al. Optoacoustic imaging of nanoparticle labeled breast cancer cells: A molecular based approach for imaging of deep tumors. Lasers in Surgery and Medicine. 2004; S16:52-52.

114. Yang XM, et al. Photoacoustic tomography of a rat cerebral cortex in vivo with au nanocages as an optical contrast agent. Nano Letters. 2007; 7:3798-3802. [PubMed: 18020475]

115. Pan DPJ, et al. Near infrared photoacoustic detection of sentinel lymph nodes with gold nanobeacons. Biomaterials. 2010; 31:4088-4093. [PubMed: 20172607]

116. Song KH, et al. Near-Infrared Gold Nanocages as a New Class of Tracers for Photoacoustic Sentinel Lymph Node Mapping on a Rat Model. Nano Letters. 2009; 9:183-188. [PubMed: 19072058]

117. Pan DPJ, et al. Photoacoustic Sentinel Lymph Node Imaging with Self-Assembled Copper Neodecanoate Nanoparticles. Acs Nano. 2012; 6:1260-1267. [PubMed: 22229462]

118. Zhang L, et al. Nanoparticles in medicine: Therapeutic applications and developments. Clinical Pharmacology \& Therapeutics. 2008; 83:761-769. [PubMed: 17957183]

119. Desai N. Challenges in development of nanoparticle-based therapeutics. AAPS J. 2012; 14:282295. [PubMed: 22407288]

120. Razansky D, et al. Multispectral opto-acoustic tomography of deep-seated fluorescent proteins in vivo. Nature Photonics. 2009; 3:412-417.

121. Razansky D, Vinegoni C, Ntziachristos V. Imaging of mesoscopic-scale organisms using selective-plane optoacoustic tomography. Physics in Medicine and Biology. 2009; 54:27692777. [PubMed: 19369709]

122. Filonov GS, et al. Deep-tissue photoacoustic tomography of a genetically encoded near-infrared fluorescent probe. Angewandte Chemie. 2012; 51:1448-1451. [PubMed: 22213541]

123. Li L, et al. Photoacoustic imaging of lacZ gene expression in vivo. Journal of Biomedical Optics. 2007; 12:020504. [PubMed: 17477703]

124. Li L, et al. Simultaneous imaging of a lacZ-marked tumor and microvasculature morphology in vivo by dual-wavelength photoacoustic microscopy. J Innov Opt Health Sci. 2008; 1:207-215. [PubMed: 19946613]

125. Cai X, et al. Multi-Scale Molecular Photoacoustic Tomography of Gene Expression. Plos One. 2012; 7:e43999. [PubMed: 22952846] 
126. Krumholz A, et al. Photoacoustic microscopy of tyrosinase reporter gene in vivo. Journal of Biomedical Optics. 2011; 16:080503. [PubMed: 21895303]

127. Laufer, J., et al. In vivo photoacoustic imaging of tyrosinase expressing tumours in mice. SPIE; San Francisco, California, USA: 2012.

128. Paproski RJ, et al. Tyrosinase as a dual reporter gene for both photoacoustic and magnetic resonance imaging. Biomedical Optics Express. 2011; 2:771-780. [PubMed: 21483602]

129. Hanahan D, Weinberg RA. Hallmarks of Cancer: The Next Generation. Cell. 2011; 144:646-674. [PubMed: 21376230]

130. Zhang HF, et al. Imaging of hemoglobin oxygen saturation variations in single vessels in vivo using photoacoustic microscopy. Applied Physics Letters. 2007; 90:053901.

131. Maslov K, Zhang HF, Wang LV. Effects of wavelength-dependent fluence attenuation on the noninvasive photoacoustic imaging of hemoglobin oxygen saturation in subcutaneous vasculature in vivo. Inverse Problems. 2007; 23:S113-S122.

132. $\mathrm{Hu} \mathrm{S}$, et al. Functional transcranial brain imaging by optical-resolution photoacoustic microscopy. Journal of Biomedical Optics. 2009; 14:040503. [PubMed: 19725708]

133. Hu S, Maslov K, Wang LHV. Noninvasive label-free imaging of microhemodynamics by opticalresolution photoacoustic microscopy. Optics Express. 2009; 17:7688-7693. [PubMed: 19399148]

134. Hu S, Maslov K, Wang LHV. In vivo functional chronic imaging of a small animal model using optical-resolution photoacoustic microscopy. Medical Physics. 2009; 36:2320-2323. [PubMed: 19610320]

135. Hu S, et al. Label-free photoacoustic ophthalmic angiography. Optics Letters. 2010; 35:1-3. [PubMed: 20664653]

136. Wang Y, Maslov K, Wang LHV. Spectrally encoded photoacoustic microscopy using a digital mirror device. Journal of Biomedical Optics. 2012; 17:066020. [PubMed: 22734776]

137. Ranasinghesagara JC, Zemp RJ. Combined photoacoustic and oblique-incidence diffuse reflectance system for quantitative photoacoustic imaging in turbid media. Journal of Biomedical Optics. 2010; 15:046016. [PubMed: 20799818]

138. Bauer AQ, et al. Quantitative photoacoustic imaging: correcting for heterogeneous light fluence distributions using diffuse optical tomography. Journal of Biomedical Optics. 2011; 16:096016. [PubMed: 21950930]

139. Cox BT, Arridge SR, Beard PC. Estimating chromophore distributions from multiwavelength photoacoustic images. J Opt Soc Am A Opt Image Sci Vis. 2009; 26:443-455. [PubMed: 19183699]

140. Laufer J, et al. Quantitative determination of chromophore concentrations from 2D photoacoustic images using a nonlinear model-based inversion scheme. Applied Optics. 2010; 49:1219-1233. [PubMed: 20220877]

141. Guo ZJ, Hu S, Wang LHV. Calibration-free absolute quantification of optical absorption coefficients using acoustic spectra in 3D photoacoustic microscopy of biological tissue. Optics Letters. 2010; 35:2067-2069. [PubMed: 20548388]

142. Guo Z, et al. Quantitative photoacoustic microscopy of optical absorption coefficients from acoustic spectra in the optical diffusive regime. Journal of Biomedical Optics. 2012; 17:066011. [PubMed: 22734767]

143. Wang RK, An L. Doppler optical micro-angiography for volumetric imaging of vascular perfusion in vivo. Optics Express. 2009; 17:8926-8940. [PubMed: 19466142]

144. Wang RK, Hurst S. Mapping of cerebro-vascular blood perfusion in mice with skin and skull intact by Optical Micro-AngioGraphy at 1.3 mu m wavelength. Optics Express. 2007; 15:1140211412. [PubMed: 19547498]

145. An L, Qin J, Wang RK. Ultrahigh sensitive optical microangiography for in vivo imaging of microcirculations within human skin tissue beds. Optics Express. 2010; 18:8220-8228. [PubMed: 20588668]

146. An L, Wang RK. In vivo volumetric imaging of vascular perfusion within human retina and choroids with optical micro-angiography. Optics Express. 2008; 16:11438-11452. [PubMed: 18648464] 
147. Cobbold, RSC. Foundations of biomedical ultrasound. Vol. xix. Oxford University Press; Oxford ; New York: 2007. p. 802

148. Fang H, Maslov K, Wang LV. Photoacoustic Doppler effect from flowing small light-absorbing particles. Physical Review Letters. 2007; 99:184501. [PubMed: 17995411]

149. Fang H, Maslov K, Wang LV. Photoacoustic Doppler flow measurement in optically scattering media. Applied Physics Letters. 2007; 91:264103.

150. Sheinfeld, A.; Gilead, S.; Eyal, A. Time-resolved photoacoustic Doppler characterization of flow using pulsed excitation. SPIE; San Francisco, California, USA: 2010.

151. Chen SL, et al. Photoacoustic correlation spectroscopy and its application to low-speed flow measurement. Optics Letters. 2010; 35:1200-1202. [PubMed: 20410966]

152. Fang H, Wang LHV. M-mode photoacoustic particle flow imaging. Optics Letters. 2009; 34:671673. [PubMed: 19252588]

153. Brunker, J.; Beard, P. Pulsed photoacoustic Doppler flowmetry using a cross correlation method. SPIE; San Francisco, California, USA: 2010.

154. Yao JJ, Wang LHV. Transverse flow imaging based on photoacoustic Doppler bandwidth broadening. Journal of Biomedical Optics. 2010; 15:021304. [PubMed: 20459226]

155. Yao J, Maslov KI, Wang LV. In vivo Photoacoustic Tomography of Total Blood Flow and Potential Imaging of Cancer Angiogenesis and Hypermetabolism. Technology in cancer research \& treatment. 2012

156. Sheinfeld A, Eyal A. Photoacoustic thermal diffusion flowmetry. Biomedical Optics Express. 2012; 3:800-813. [PubMed: 22574267]

157. Beard P. Biomedical photoacoustic imaging. Interface Focus. 2011; 1:602-631. [PubMed: 22866233]

158. Wei C, et al. Photoacoustic flow measurements based on wash-in analysis of gold nanorods. IEEE Transactions on Ultrasonics Ferroelectrics and Frequency Control. 2007; 54:1131-1141.

159. Wei CW, et al. Photoacoustic flow measurements with gold nanoparticles. IEEE Transactions on Ultrasonics Ferroelectrics and Frequency Control. 2006; 53:1955-1959.

160. Song L, Maslov K, Wang LV. Section-illumination photoacoustic microscopy for dynamic 3D imaging of microcirculation in vivo. Optics Letters. 2010; 35:1482-1484. [PubMed: 20436610]

161. Hanahan D, Weinberg RA. The hallmarks of cancer. Cell. 2000; 100:57-70. [PubMed: 10647931]

162. Liu T, et al. Combined photoacoustic microscopy and optical coherence tomography can measure metabolic rate of oxygen. Biomedical Optics Express. 2011; 2:1359-1365. [PubMed: 21559147]

163. Jiang Y, et al. Blood oxygen flux estimation with a combined photoacoustic and high-frequency ultrasound microscopy system: a phantom study. Journal of Biomedical Optics. 2012; 17:036012. [PubMed: 22502570]

164. Wang Y, Wang LV. Förster resonance energy transfer photoacoustic microscopy. Journal of Biomedical Optics. 2012; 17:086007. [PubMed: 23224194]

165. Danielli A, et al. Single-wavelength functional photoacoustic microscopy in biological tissue. Optics Letters. 2011; 36:769-771. [PubMed: 21368977]

166. Hu, S., et al. Dichroism optical-resolution photoacoustic microscopy. SPIE; San Francisco, California, USA: 2012.

167. Jin YD, et al. Multifunctional nanoparticles as coupled contrast agents. Nature Communications. 2010; 1:1042.

168. Xia J, et al. Suppression of background signal in magnetomotive photoacoustic imaging of magnetic microspheres mimicking targeted cells. Journal of Biomedical Optics. 2012; 17:061224. [PubMed: 22734754]

169. Moscoso M, Keller JB, Papanicolaou G. Depolarization and blurring of optical images by biological tissue. J Opt Soc Am A Opt Image Sci Vis. 2001; 18:948-960. [PubMed: 11318346]

170. Ray A, et al. Lifetime-based photoacoustic oxygen sensing in vivo. Journal of Biomedical Optics. 2012; 17:057004. [PubMed: 22612143]

171. Chatni MR, et al. Functional photoacoustic microscopy of pH. Journal of Biomedical Optics. 2011; 16:100503. [PubMed: 22029342] 
172. Ashkenazi S, et al. Photoacoustic probing of fluorophore excited state lifetime with application to oxygen sensing. Journal of Biomedical Optics. 2008; 13:034023. [PubMed: 18601568]

173. Ashkenazi S. Photoacoustic lifetime imaging of dissolved oxygen using methylene blue. Journal of Biomedical Optics. 2010; 15:040501. [PubMed: 20799768]

174. Oladipupo S, et al. VEGF is essential for hypoxia-inducible factor-mediated neovascularization but dispensable for endothelial sprouting. Proceedings of the National Academy of Sciences of the United States of America. 2011; 108:13264-13269. [PubMed: 21784979]

175. Oladipupo SS, et al. Conditional HIF-1 induction produces multistage neovascularization with stage-specific sensitivity to VEGFR inhibitors and myeloid cell independence. Blood. 2011; 117:4142-4153. [PubMed: 21307392]

176. Bitton R, et al. A 3-D high-frequency array based 16 channel photoacoustic microscopy system for in vivo micro-vascular imaging. IEEE Trans Med Imaging. 2009; 28:1190-1197. [PubMed: 19131292]

177. Cui HZ, Yang XM. In vivo imaging and treatment of solid tumor using integrated photoacoustic imaging and high intensity focused ultrasound system. Medical Physics. 2010; 37:4777-4781. [PubMed: 20964197]

178. de la Zerda A, et al. Ultrahigh Sensitivity Carbon Nanotube Agents for Photoacoustic Molecular Imaging in Living Mice. Nano Letters. 2010; 10:2168-2172. [PubMed: 20499887]

179. Li ML, et al. In-vivo photoacoustic microscopy of nanoshell extravasation from solid tumor vasculature. Journal of Biomedical Optics. 2009; 14:010507. [PubMed: 19256687]

180. Olafsson R, et al. Real-time, contrast enhanced photoacoustic imaging of cancer in a mouse window chamber. Optics Express. 2010; 18:18625-18632. [PubMed: 20940754]

181. Liao LD, et al. Imaging brain hemodynamic changes during rat forepaw electrical stimulation using functional photoacoustic microscopy. Neuroimage. 2010; 52:562-570. [PubMed: 20362680]

182. Tsytsarev V, et al. Photoacoustic microscopy of microvascular responses to cortical electrical stimulation. Journal of Biomedical Optics. 2011; 16:076002. [PubMed: 21806263]

183. Silverman RH, et al. High-resolution photoacoustic imaging of ocular tissues. Ultrasound Med Biol. 2010; 36:733-742. [PubMed: 20420969]

184. Zhang HF, et al. In vivo volumetric imaging of subcutaneous microvasculature by photoacoustic microscopy. Optics Express. 2006; 14:9317-9323. [PubMed: 19529315]

185. Favazza, C., et al. In vivo functional human imaging using photoacoustic microscopy: response to ischemic and thermal stimuli. SPIE; San Francisco, California, USA: 2010.

186. Favazza CP, Cornelius LA, Wang LHV. In vivo functional photoacoustic microscopy of cutaneous microvasculature in human skin. Journal of Biomedical Optics. 2011; 16:026004. [PubMed: 21361688]

187. Favazza C, et al. In vivo photoacoustic microscopy of human skin. Journal of Investigative Dermatology. 2010; 130:S145.

188. Song LA, et al. Ultrasound-array-based real-time photoacoustic microscopy of human pulsatile dynamics in vivo. Journal of Biomedical Optics. 2010; 15:021303. [PubMed: 20459225]

189. Yang, J-M., et al. A 2.5-mm outer diameter photoacoustic endoscopic mini-probe based on a highly sensitive PMN-PT ultrasonic transducer. SPIE; San Francisco, California, USA: 2012.

190. Yang, J-M., et al. Toward dual-wavelength functional photoacoustic endoscopy: laser and peripheral optical systems development. SPIE; San Francisco, California, USA: 2012.

191. Rowland KJ, et al. Immediate alterations in intestinal oxygen saturation and blood flow after massive small bowel resection as measured by photoacoustic microscopy. Journal of Pediatric Surgery. 2012; 47:1143-1149. [PubMed: 22703784]

192. Taruttis A, et al. Real-time imaging of cardiovascular dynamics and circulating gold nanorods with multispectral optoacoustic tomography. Optics Express. 2010; 18:19592-19602. [PubMed: 20940855]

193. Zemp RJ, et al. Realtime Photoacoustic Microscopy of Murine Cardiovascular Dynamics. Optics Express. 2008; 16:18551-18556. [PubMed: 18958134] 
194. Yeh CH, et al. Photoacoustic microscopy of blood pulse wave. Journal of Biomedical Optics. 2012; 17:070504. [PubMed: 22894460]

195. Ye SQ, et al. Label-free imaging of zebrafish larvae in vivo by photoacoustic microscopy. Biomedical Optics Express. 2012; 3:360-365. [PubMed: 22312588]

196. Song L, et al. High-speed dynamic 3D photoacoustic imaging of sentinel lymph node in a murine model using an ultrasound array. Medical Physics. 2009; 36:3724-3729. [PubMed: 19746805]

197. Stein EW, Maslov K, Wang LHV. Noninvasive, in vivo imaging of blood-oxygenation dynamics within the mouse brain using photoacoustic microscopy. Journal of Biomedical Optics. 2009; 14:020502. [PubMed: 19405708]

198. Zhang HF, et al. Collecting back-reflected photons in photoacoustic microscopy. Optics Express. 2010; 18:1278-1282. [PubMed: 20173952]

199. Zhang X, et al. Simultaneous dual molecular contrasts provided by the absorbed photons in photoacoustic microscopy. Opt. Lett. 2010; 35:4018-4020. [PubMed: 21124598]

200. Wang Y, et al. Integrated Photoacoustic and Fluorescence Confocal Microscopy. IEEE Transactions on Biomedical Engineering. 2010; 57:2576-2578. [PubMed: 20639165]

201. Li L, et al. Three-dimensional combined photoacoustic and optical coherence microscopy for in vivo microcirculation studies. Optics Express. 2009; 17:16450-16455. [PubMed: 19770860]

202. van Raaij, ME., et al. Femtosecond photoacoustics: integrated two-photon fluorescence and photoacoustic microscopy. SPIE; San Francisco, California, USA: 2010.

203. Harrison, T., et al. Fast-scanning ultrasonic-photoacoustic biomicroscope: in vivo performance. SPIE; San Francisco, California, USA: 2010.

204. Niederhauser JJ, et al. Combined ultrasound and optoacoustic system for real-time high-contrast vascular imaging in vivo. IEEE Transactions on Medical Imaging. 2005; 24:436-440. [PubMed: 15822801]

205. Li WY, et al. Gold nanocages covered with thermally-responsive polymers for controlled release by high-intensity focused ultrasound. Nanoscale. 2011; 3:1724-1730. [PubMed: 21321760]

206. Akers WJ, et al. Noninvasive photoacoustic and fluorescence sentinel lymph node identification using dye-loaded perfluorocarbon nanoparticles. Acs Nano. 2011; 5:173-182. [PubMed: 21171567]

207. Yang JM, et al. Photoacoustic endoscopy. Optics Letters. 2009; 34:1591-1593. [PubMed: 19448831]

208. Wei W, et al. Integrated ultrasound and photoacoustic probe for co-registered intravascular imaging. Journal of Biomedical Optics. 2011; 16:106001. [PubMed: 22029348]

209. Karpiouk AB, Wang B, Emelianov SY. Development of a catheter for combined intravascular ultrasound and photoacoustic imaging. Review of Scientific Instruments. 2010; 81:014901. [PubMed: 20113121]

210. Yang JM, et al. Simultaneous functional photoacoustic and ultrasonic endoscopy of internal organs in vivo. Nature Medicine. 2012; 18:1297-1303.

211. Yang J-M, et al. A 2.5-mm diameter probe for photoacoustic and ultrasonic endoscopy. Opt. Express. 2012; 20:23944-23953. [PubMed: 23188360]

212. Zhang, EZ.; Beard, PC. A miniature all-optical photoacoustic imaging probe. SPIE; San Francisco, California, USA: 2011.

213. Hasegawa N, et al. Preoperative staging of superficial esophageal carcinoma: comparison of an ultrasound probe and standard endoscopic ultrasonography. Gastrointestinal Endoscopy. 1996; 44:388-93. [PubMed: 8905355] 
(a)

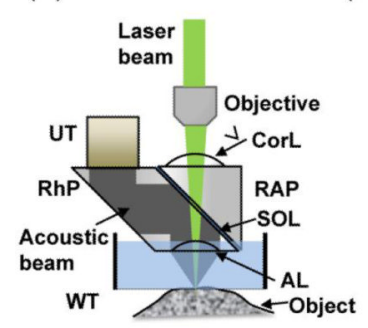

(b)

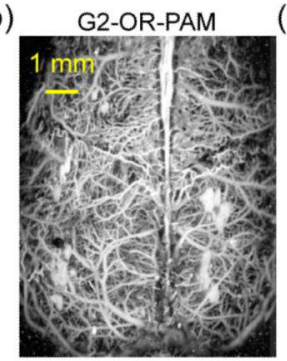

(c)

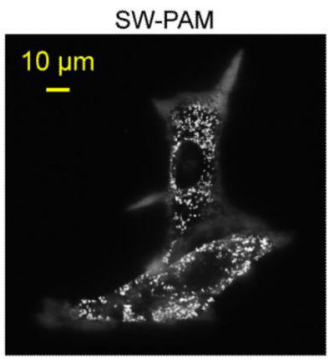

(d)

(e)

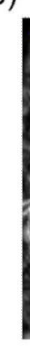

AR-PAM

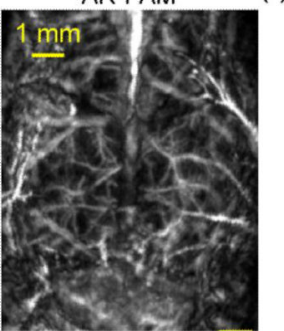

(f)

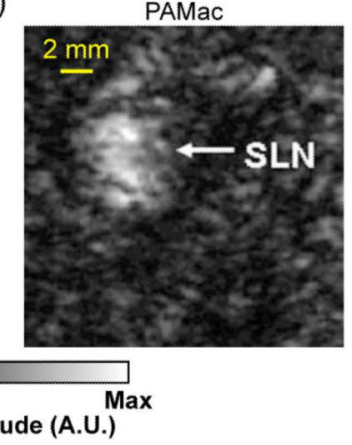

Figure 1.

Representative photoacoustic microscopy. (a) Second-generation optical-resolution photoacoustic microscopy (G2-OR-PAM), where the lateral resolution is determined by the diffraction-limited optical focusing. AL, acoustic lens; Corl, correction lens; RAP, right angled prism; RhP, rhomboid prism; SOL, silicone oil layer; UT, ultrasonic transducer; WT, water tank. (b) G2-OR-PAM of cortical vasculature in a living mouse with the scalp removed but the skull intact. (c) Sub-wavelength photoacoustic microscopy (SW-PAM) of a melanoma cell, where single melanosomes can be resolved. Reprinted with permission from [35]. (d) Dark-field acoustic-resolution photoacoustic microscopy (AR-PAM), where the lateral resolution is determined by the diffraction-limited acoustic focusing. (e) Dark-field AR-PAM of the cortical vasculature in a living mouse with both the scalp and skull intact. Reprinted with permission from [43]. (f) Deep photoacoustic macroscopy (PAMac) of the sentinel lymph node (SLN) in a living rat. The SLN was about $18 \mathrm{~mm}$ deep. Reprinted with permission from [47]. 
(a)

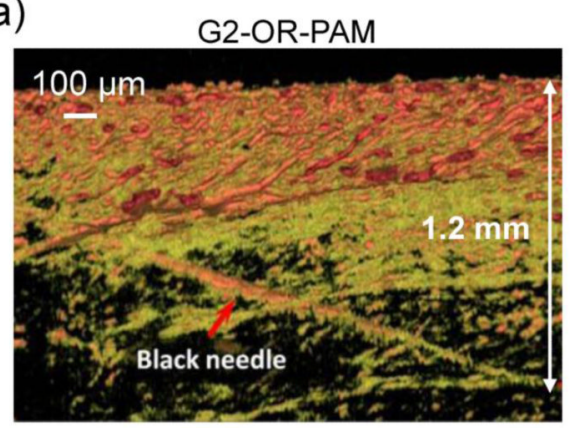

Min (b)

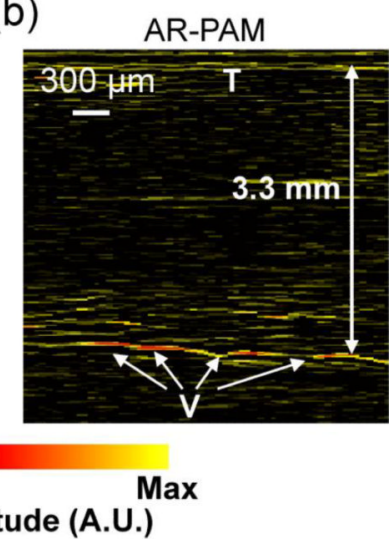

Figure 2.

Representative maximum imaging depths of PAM. (a) G2-OR-PAM of a black needle obliquely inserted into the leg of an anesthetized living mouse. A maximum imaging depth of $\sim 1.2 \mathrm{~mm}$ was achieved. Reprinted with permission from [38]. (b) Dark-field AR-PAM of subcutaneous vessels in a living rat when the skin was covered by a chicken breast tissue slab. A maximum imaging depth of $\sim 3.3 \mathrm{~mm}$ was achieved. V, vessels; T, top surface of the chicken tissue. Reprinted with permission from [33]. 


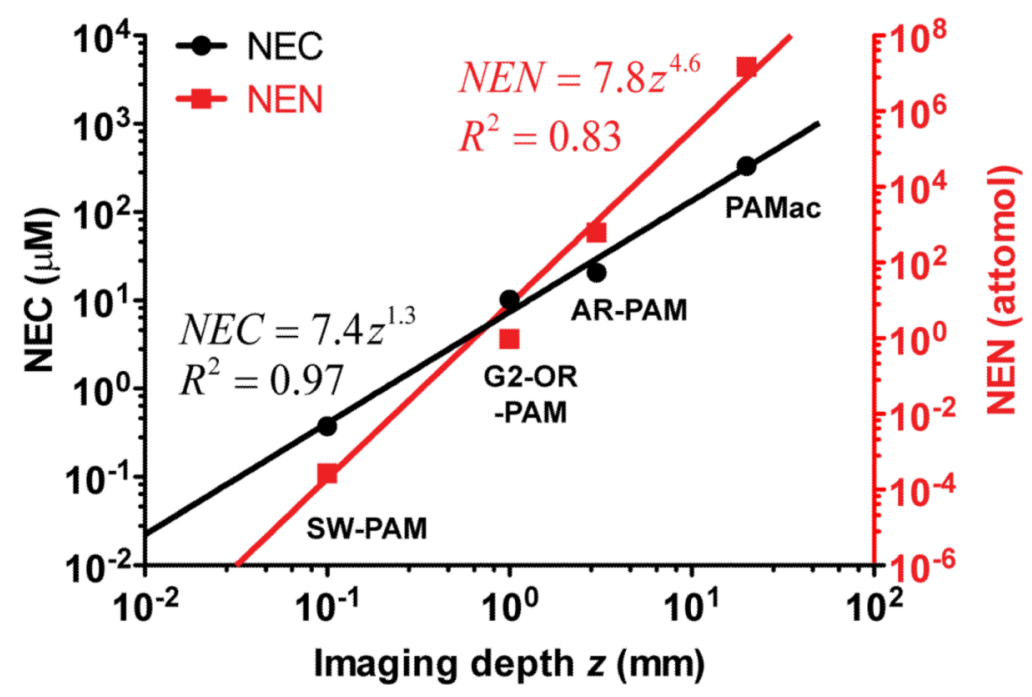

Figure 3.

Noise-equivalent molar concentration (NEC, left axis, black circles) of hemoglobin molecules and noise-equivalent number (NEN, right axis, red squares) of hemoglobin molecules per resolution voxel versus the imaging depth of different photoacoustic imaging systems. The results were quantified from the reported in vivo data in the literature. The solid curves are power function fittings. 


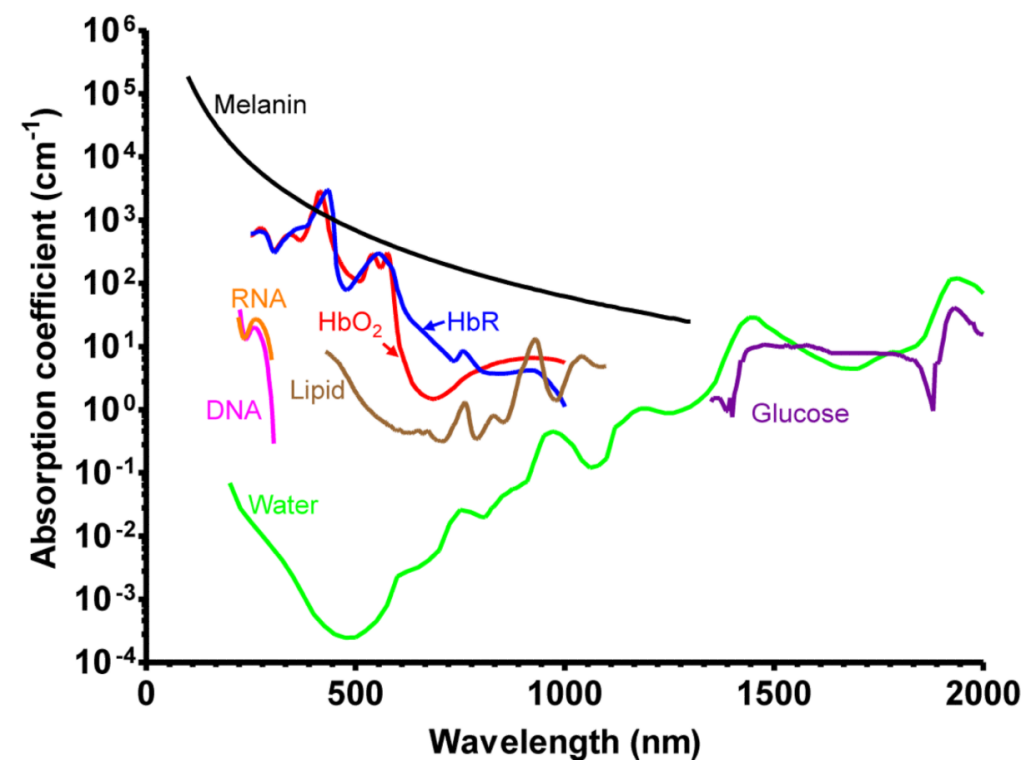

Figure 4.

Absorption spectra of major endogenous contrast agents in biological tissue. Oxyhemoglobin, red line (150 g/L in blood); Deoxy-hemoglobin, blue line (150 g/L in blood); Lipid, brown line (20\% by volume in tissue); Water, green line ( $80 \%$ by volume in tissue); DNA, magenta line (1 g/L in cell nuclei); RNA, orange line (1 g/L in cell nuclei); Melanin, black line (14.3 g/L in medium human skin); Glucose, purple line (720 mg/L in blood). 
(a)

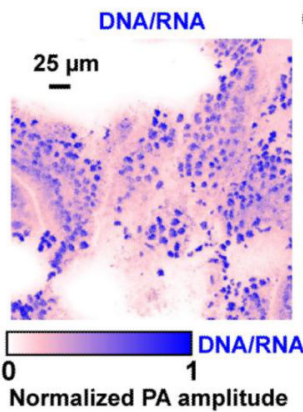

(d)

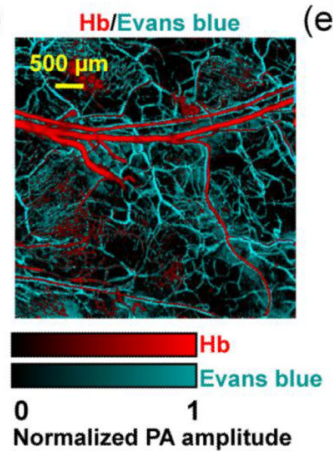

(b)

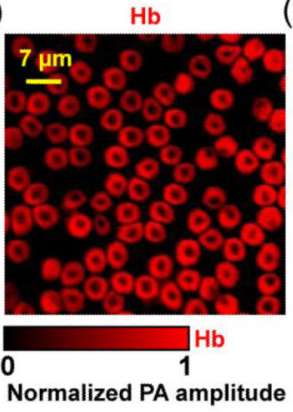

(e)

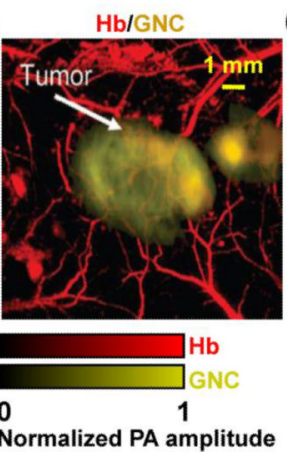

(c)

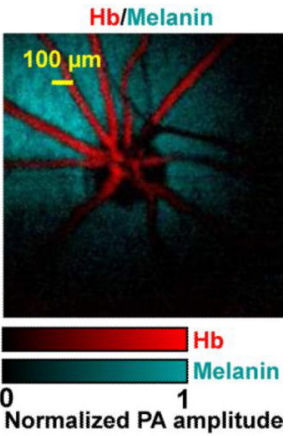

(f)

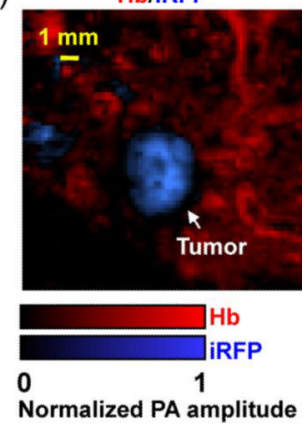

Figure 5.

Representative multi-contrast PAM images. (a) UV-PAM of cell nuclei (shown in blue) in a $6-\mu \mathrm{m}$-thick slice from mouse small intestine. No histology staining was needed here. The signals of cell nuclei come from DNA and RNA. Reprinted with permission from [39]. (b) SW-PAM of red blood cells (RBC). The signals of RBCs come from hemoglobin (Hb). Reprinted with permission from [35]. (c) Photoacoustic ophthalmoscopy of retinal vessels (shown in red) and the retinal pigment epithelium layer (RPE, shown in green) in a living rat. The signals of the RPE layer come from melanin. Reprinted with permission from [68]. (d) OR-PAM of the microvasculature in a mouse ear, where the capillaries (shown in green) were enhanced by Evans blue dye. Reprinted with permission from [101]. (e) AR-PAM of a subcutaneously inoculated B16 melanoma labeled with targeted gold nanocages (GNC, shown in yellow) and the surrounding vessels (shown in red) in a living mouse. Reprinted with permission from [112]. (f) PAMac of a mouse mammary gland tumor which expressed a near-infrared fluorescent protein iRFP (shown in blue). Reprinted with permission from [122]. 

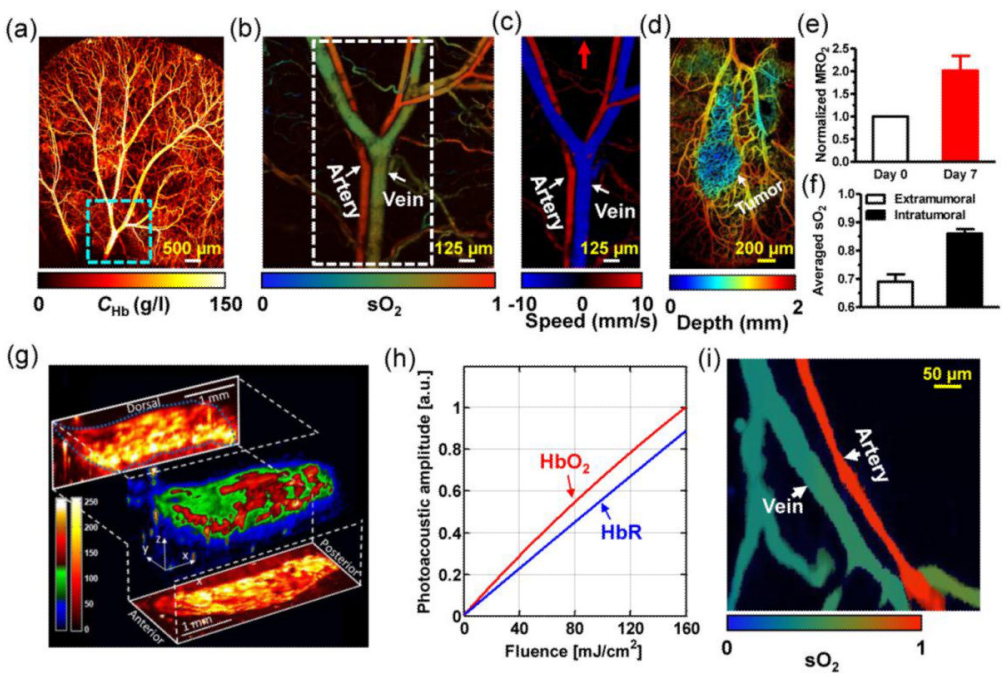

Figure 6.

Representative multi-parameter PAM images. (a) OR-PAM of the total concentration of hemoglobin $\left(\mathrm{C}_{\mathrm{Hb}}\right)$ in a mouse ear. (b) OR-PAM of the oxygen saturation of hemoglobin $\left(\mathrm{sO}_{2}\right)$ in the area indicated by the dashed box in (a). (c) OR-PAM of blood flow in the area indicated by the dashed box in (b). Red arrow: positive flow direction. (d) OR-PAM of a mouse ear bearing a xenographed glioblastoma on Day 7. The vasculature of the mouse ear was color-coded by depth: blue (superficial) to red (deep). (e) $\mathrm{MRO}_{2}$ change induced by tumor growth in (d). A $100 \%$ increase in $\mathrm{MRO}_{2}$ indicated the tumor hypermetabolism. (f) Averaged $\mathrm{sO}_{2}$ inside and outside the tumor, which suggested that the tumor region was hyperoxic. (a-f) were adapted with permission from [82]. (g) Vibrational PAM of fat bodies in a 3rd-instar larva of Drosophila melanogaster embedded in a thin layer of agar gel. The PA signals come from the overtone absorption of $\mathrm{CH}$ bond stretch in lipid. Reprinted with permission from [95]. (h) OR-PAM measurement of the absorption relaxation time of oxyhemoglobin $\left(\mathrm{HbO}_{2}\right)$ and deoxy-hemoglobin $(\mathrm{HbR})$, made by fitting the PA signal amplitude as a function of incident laser fluence. Reprinted with permission from [60]. (i) Singlewavelength PAM measurement of the $\mathrm{sO}_{2}$ in a mouse ear, based on the relaxation times of $\mathrm{HbO}_{2}$ and $\mathrm{HbR}$. Reprinted with permission from [165]. 
(a)

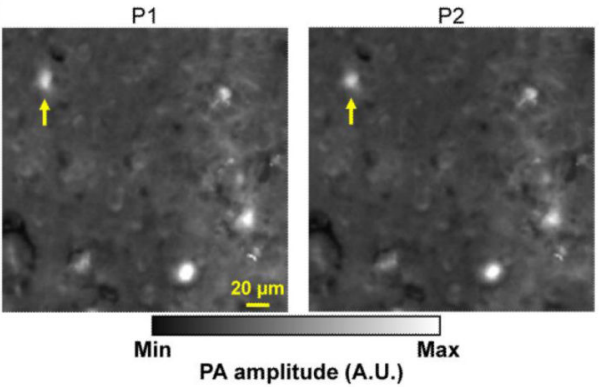

(c)

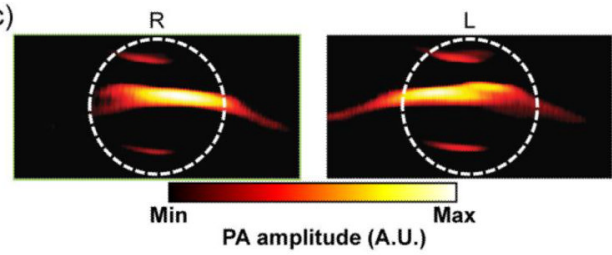

(b)

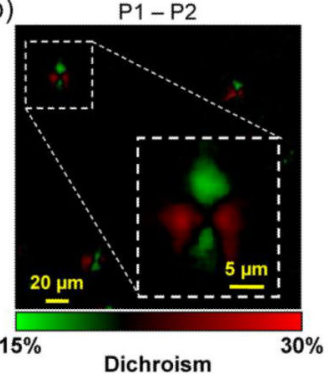

(d)

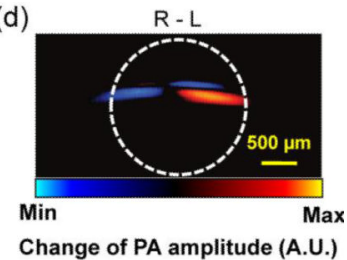

Figure 7.

PAM specificity enhancement. (a) Dichroism PAM of amyloid plaques in an APP/PS1 mouse brain section stained with Congo Red (CR), acquired with two orthogonally polarized optical irradiations P1 and P2. (b) Subtraction of P1 and P2, which eliminates the nondichroic background and highlights the dichroic contrast of the amyloid plaques. Inset: close-up of the boxed area, showing the bipolar dichroism pattern of a representative amyloid plaque indicated by yellow arrows in $(a-b)$. $(a-b)$ were adapted with permission from [166]. (c) Magnetomotive PAM of magnetic nanoparticles (MNP) trapped in MNP-ink solution in a tube. R: the tube was closer to the right magnet; $\mathrm{L}$ : the tube was closer to the left magnet. Note that MNPs changed their positions while the nonmagnectic ink did not. The white dashed circles indicate the tube boundary. (d) Subtraction of R and L, which substantially suppresses the undesired background signals. (c-d) were adapted with permission from [168]. 

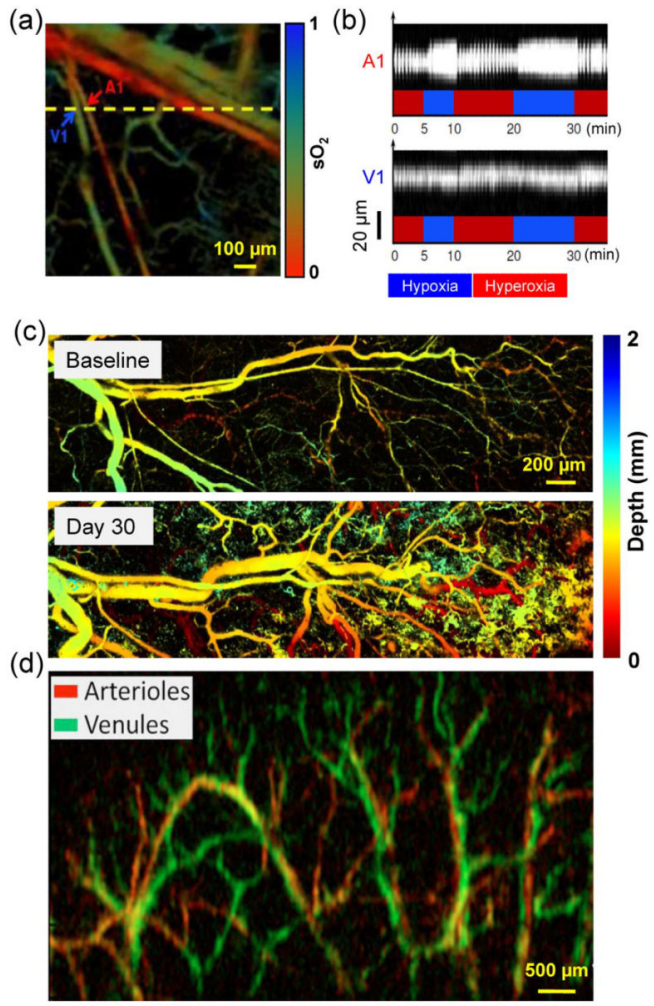

Figure 8.

Representative PAM applications in preclinical studies. (a) OR-PAM of the $\mathrm{sO}_{2}$ in a mouse ear where an arteriole-venule pair ( $20 \mu \mathrm{m}$ in diameter) was chosen for vasodilation and vasomotion study. A1, a representative arteriole; V1, a representative venule. (b) Vasodilation and vasomotion in response to switching the physiological state between systemic hyperoxia and hypoxia: a B-scan monitoring of the changes in the cross-sections of the arteriole and venule. The vasomotion of the arteriole had greater amplitude than that of the venule but the same frequency. (a-b) were adapted with permission from [133]. (c) Chronic OR-PAM of the neovascularization in the same mouse. The blood vessels of the mouse ear were color-coded by depth: blue (superficial) to red (deep). Adapted with permission from [175]. (d) Linear-array PAM of wash-in dynamics of Evans blue in a mouse ear. The whole dynamic flow of Evans blue from arteries (shown in red) to veins (shown in green) was captured. Reprinted with permission from [160]. 
Figure 9. (a)

(b)

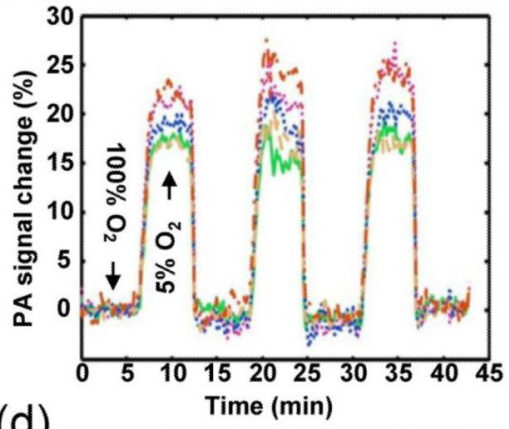

(c)

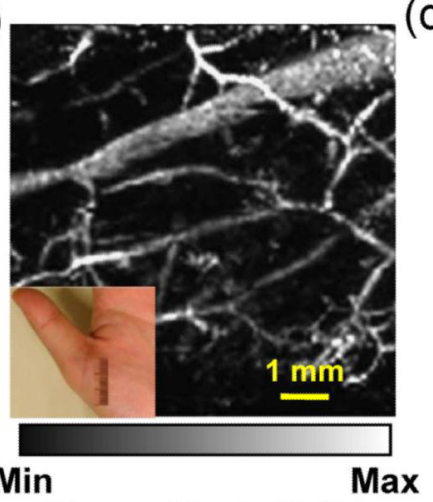

PA amplitude (A.U.)

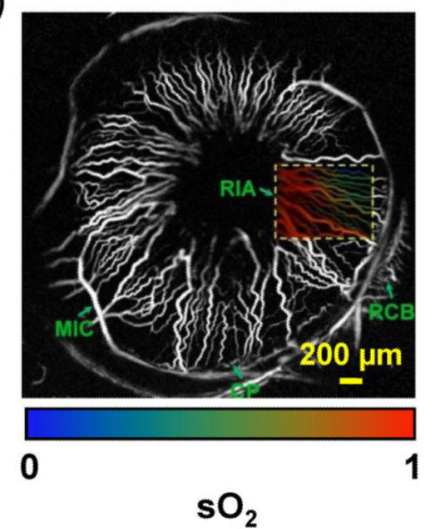

Representative PAM applications in preclinical and human studies. (a) AR-PAM of cortical vasculature in a living mouse. Dotted white line indicates the line scanning range. CS, coronal suture. (b) Dynamic vessel response profiles acquired through a hypoxic challenge and shown in percent change of ratiometric PA signals at two wavelengths of $561 \mathrm{~nm}$ and $570 \mathrm{~nm}$. Each colored trace corresponds to the respective cortical vessel crossed by the dotted line in (a). (a-b) were adapted with permission from [197]. (c) AR-PAM of subcutaneous vasculature of the palm of a human hand at $584 \mathrm{~nm}$, with an incident laser fluence of $\sim 6 \mathrm{~mJ} / \mathrm{cm}^{2}$. Inset: photograph of the imaged area. Reprinted with permission from [33]. (d) OR-PAM of the iris microvasculature in a living adult mouse. A vessel-by-vessel $\mathrm{sO}_{2}$ mapping (shown in color scale) was generated and overlaid on the vascular image acquired at $570 \mathrm{~nm}$ (shown in gray scale). $\mathrm{CP}$, ciliary process; MIC, major iris circle; RCB, recurrent choroidal branch; RIA, radial iris artery. Reprinted with permission from [135]. 


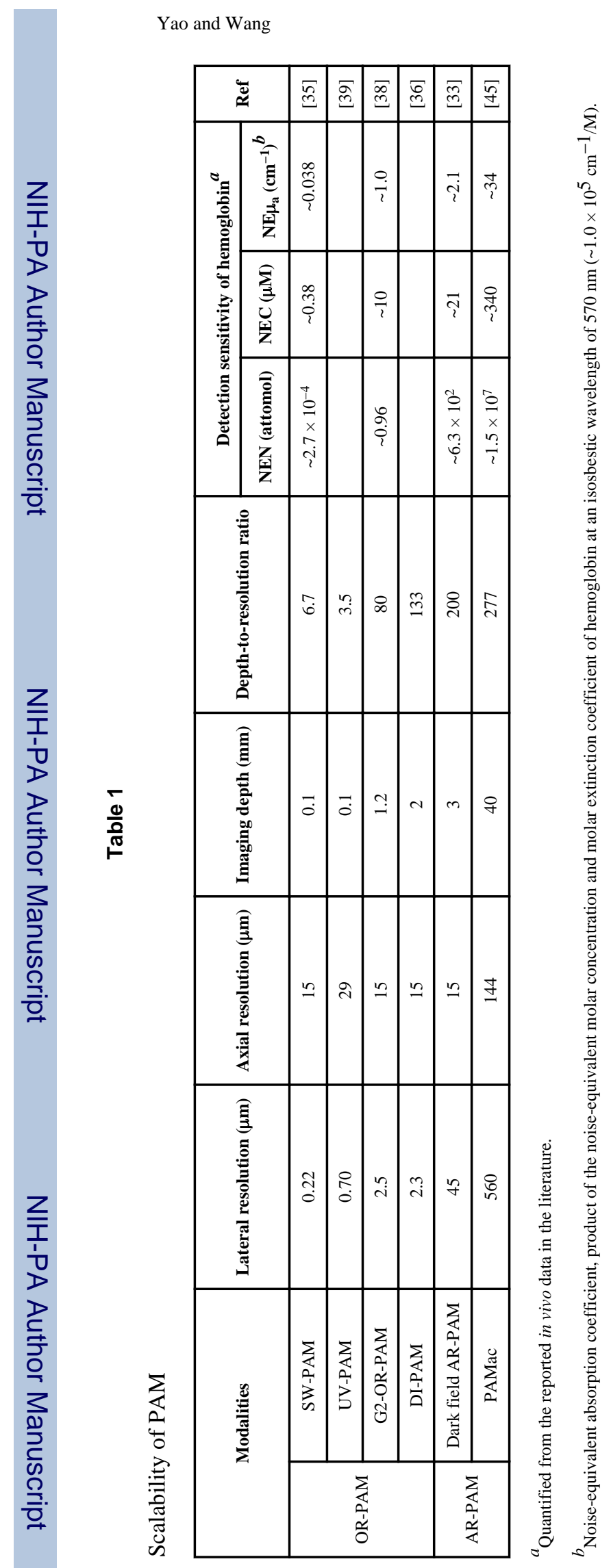

Page 34

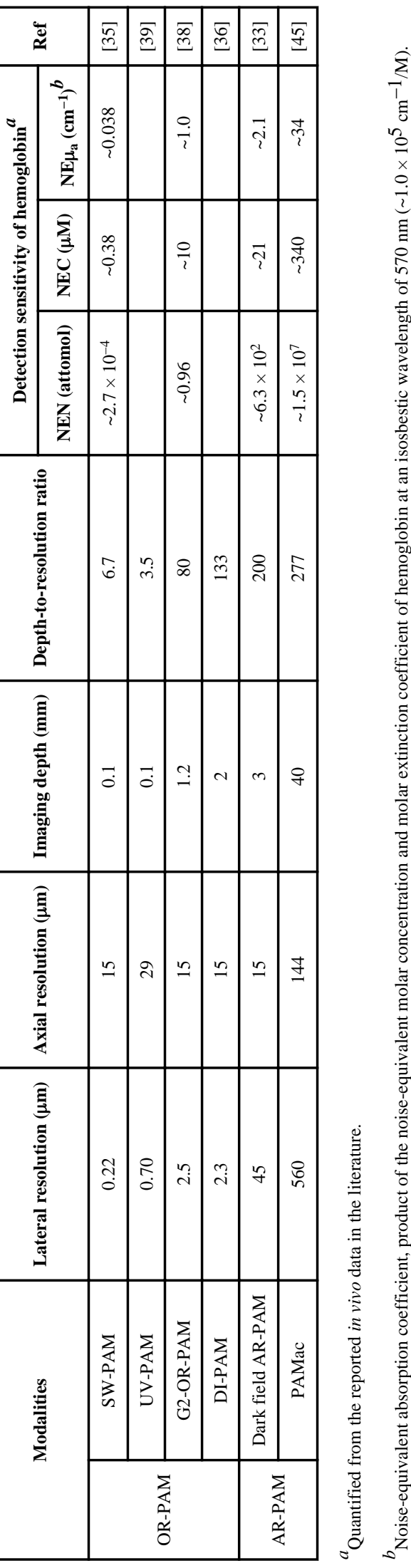

Laser Photon Rev. Author manuscript; available in PMC 2014 January 10. 


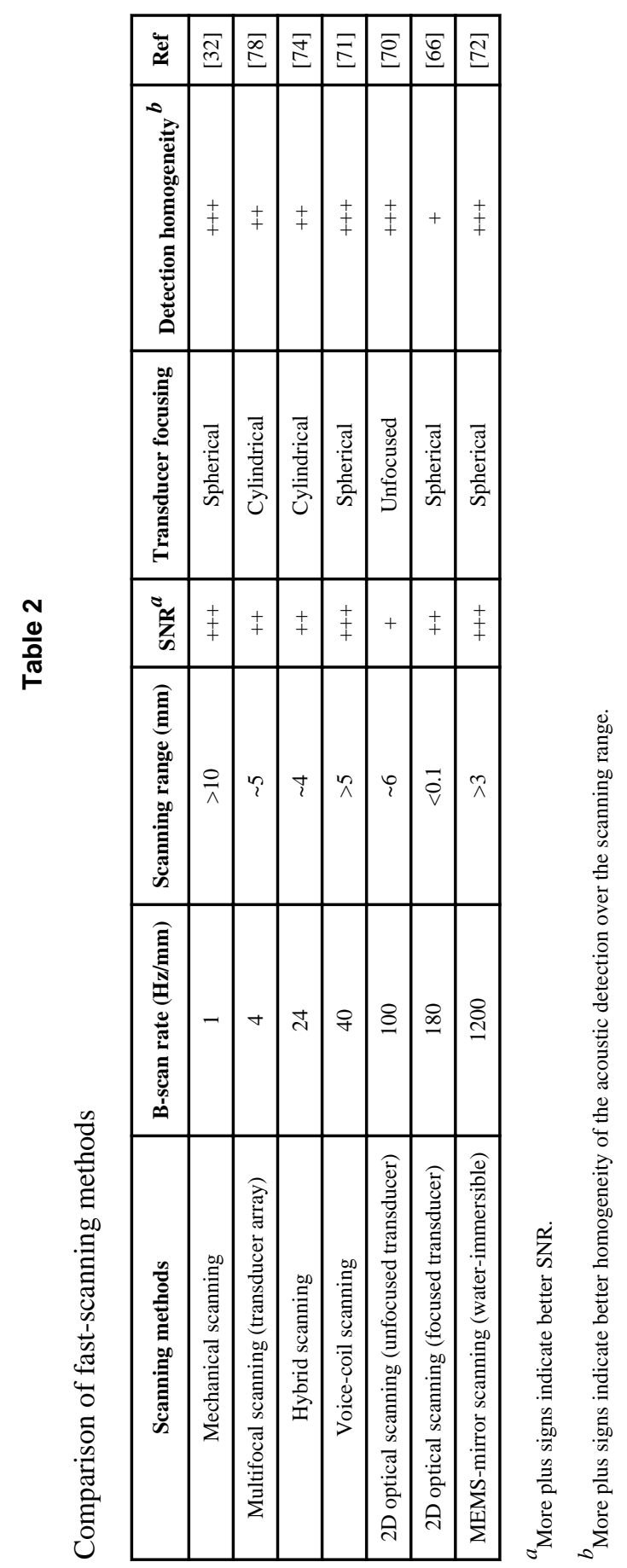

Laser Photon Rev. Author manuscript; available in PMC 2014 January 10. 
Table 3

Parameters measured by PAM

\begin{tabular}{|c|c|c|c|c|}
\hline Parameters & Endogenous contrast & Absolute unit & Representative applications & Ref \\
\hline $\mathrm{C}_{\mathrm{Hb}}$ & $\sqrt{ }$ & $\sqrt{ }$ & Imaging blood perfusion in angiogenesis & [141] \\
\hline $\mathrm{sO}_{2}$ & $\sqrt{ }$ & $\sqrt{ }$ & $\begin{array}{l}\text { Imaging blood oxygenation in tumor } \\
\text { microenvironment }\end{array}$ & [130] \\
\hline $\mathrm{pO}_{2}$ & & & Imaging oxygenation of solid tumors & [170] \\
\hline Flow speed & $\sqrt{ }$ & $\sqrt{ }$ & Imaging microcirculation diseases & [61] \\
\hline $\mathrm{MRO}_{2}$ & $\sqrt{ }$ & $\sqrt{ }$ & Imaging hypermetabolism of cancers & [82] \\
\hline $\begin{array}{c}\text { Overtone absorption of chemical } \\
\text { bond }\end{array}$ & $\sqrt{ }$ & & Diagnosing atherosclerosis & [95] \\
\hline Raman scattering & $\sqrt{ }$ & & Imaging and chemical analysis of microcalcifications & [96] \\
\hline Dichroism & & & Imaging amyloid plaques in Alzheimer's disease & [166] \\
\hline Magnetomotion & & & Detecting circulating cancer cells & {$[168]$} \\
\hline Absorption relaxation time & $\sqrt{ }$ & $\sqrt{ }$ & $\begin{array}{c}\text { Single-wavelength measurement of blood } \\
\text { oxygenation }\end{array}$ & [60] \\
\hline $\mathrm{pH}$ & & $\sqrt{ }$ & Diagnosing metabolic diseases such as diabetes & [171] \\
\hline
\end{tabular}

